\title{
Research Paper \\ The Effect of a Death Anxiety Therapeutic Package Based on Acceptance and Commitment Therapy on Death Avoidance, Mental Health and Quality of Life of Cancer Patients
}

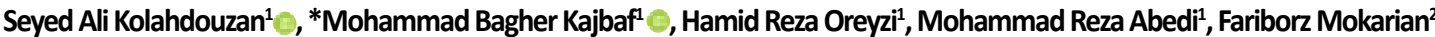

1. Department of Psychology, Faculty of Education and Psychology, University of Isfahan, Isfahan, Iran.

2. Department of Internal Medicine, School of Medicine, Isfahan University of Medical Sciences, Isfahan, Iran.

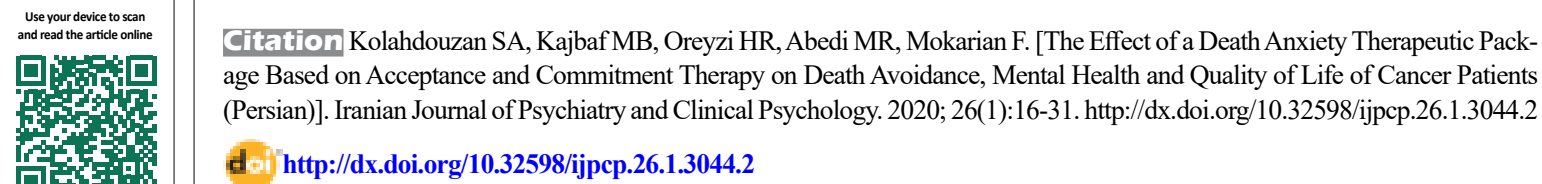
age Based on Acceptance and Commitment Therapy on Death Avoidance, Mental Health and Quality of Life of Cancer Patients (Persian)]. Iranian Journal of Psychiatry and Clinical Psychology. 2020; 26(1):16-31. http://dx.doi.org/10.32598/ijpcp.26.1.3044.2

http://dx.doi.org/10.32598/ijpcp.26.1.3044.2

Key words:

Acceptance and commitment therapy, Death anxiety, Death avoidance, Mental health, Quality of life, Cancer

\begin{abstract}
A B S TRACT
Objectives The purpose of this study was to evaluate the effect of a death anxiety therapeutic package designed based on Acceptance and Commitment Therapy (ACT) on death avoidance, mental health, and quality of life of cancer patients.

Methods This parallel-experimental study was conducted on 28 cancer patients ( 19 females and 9 males aged 26-71 years) referred to Seyedoshohada Hospital in Isfahan, Iran. They were randomly assigned into two groups of intervention $(n=14)$ and control $(n=14)$. The death anxiety package was designed in the University of Nevada and Lawshe's method was used for examining its content validity ratio. The intervention group received therapy at seven sessions, once a week, while the control group received simple writing exercises with no intervention. Participants completed World Health Organization Quality of Life-BREF ( $\alpha=0.91$ ), Death Attitude Profile-Revised $(\alpha=0.88)$, and Acceptance and Action Questionnaire $(\alpha=0.87)$ three times as pretest, posttest, and follow-up assessments. Data were analyzed by ANCOVA in SPSS V. 21 software.

Results The intervention group showed a significant decrease in death attitude dimensions of "fear of death" and "death avoidance" and a significant increase in the quality of life dimension of "mental health" compared to the control group.

Conclusion The designed package based on ACT seems to reduce the death anxiety and death avoidance, and any change in attitude towards death in cancer patients may be related to their mental health.
\end{abstract}

\section{Extended Abstract}

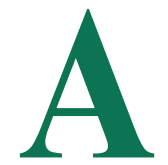

\section{Introduction}

ccording to the International Agency for Research on Cancer report in 2018, it is predicted that the rate of increase in the number of cancer patients in Iran will reach 35\% until 2025 [1]. One of psychological problems that the cancer patients have to deal with, par- ticularly during metastasis, is death anxiety. The purpose of Acceptance and Commitment Therapy (ACT) is to help patients replace the constructive behaviors with dysfunctional and problematic behaviors with no goal to change the intensity or frequency of negative emotions [10].

Studies conducted in foreign countries have reported the positive impact of acceptance strategies [13-15], living a value-based life [16], and psychological flexibility [17-

* Corresponding Author:

Mohammad Bagher Kajbaf, PhD.

Address: Department of Psychology, Faculty of Education and Psychology, University of Isfahan, Isfahan, Iran.

Tel: +98 (31) 37935465

E-mail: m.b.kaj@edu.ui.ac.ir 
20] on mental health and quality of life of cancer patients. Similar studies in Iran have also reported the significant effect of ACT therapy on decreasing death anxiety (in HIV samples) [21], anxiety symptoms [26-29], depression [30-33], stress [34], anxiety-related pain [35], and quality of life [22-25] of cancer patients.

\section{Method}

\section{Package design}

This package is designed based on ACT and mainly focuses on dealing with 'death anxiety' in cancer patients. This package was prepared by the researcher $(\mathrm{a} \mathrm{PhD}$. candidate from Isfahan University) and under the supervision of Dr. Steven Hayes in the department of psychology at the University of Nevada. The researcher participated in several seminars, workshops, and projects in Iran and United States to learn ACT theory and practice. He interviewed 12 psychologists and psychiatrists at the University of Nevada discussing the package design and possible paragraphs. The initial design of package was developed with 77 written paragraphs. Dr. Hayes and seven $\mathrm{PhD}$ students at the University of Nevada examined and confirmed the consistency of paragraphs with the main ACT paragraphs. In the end, the paragraphs with a Content Validity Ratio (CVR) of less than 0.62 were removed and 60 approved paragraphs were remained.

\section{Package implementation}

The study was implemented on 28 cancer patients (19 females and 9 males aged 26-71 years). The inclusion criteria were: having at least 18 years old, not being under any other psychological treatments, having reading and writing ability, ability to be present physically in the sessions, having consent for participating in the sessions, and having an introduction letter from Seyedoshohada Hospital in Isfahan. After selecting participants based on their medical records, they were randomly assigned to intervention $(n=14)$ and control $(n=14)$ groups. It should be mentioned that two participants were excluded from the study due to hospitalization and traveling, and the final sample size was then reduced to 26 .

The intervention group received therapy at 7 sessions, once a week, based on the designed ACT package with a focus on attitude towards death, while the control group received some writing exercises as placebo. World Health Organization Quality of Life-BREF, Death Attitude Profile-Revised, and Acceptance and Action Questionnaire were used as data collection tools before intervention, after intervention, and in a two-month follow-up period. MANOVA was used for data analysis in SPSS V. 21 software.

\section{Results}

The MANOVA results showed that the intervention and control groups were meaningfully different in terms of fear of death, death avoidance and mental health $(\mathrm{P}<0.001)$, but

Table 1. Mean scores at three time intervals in experimental and control groups

\begin{tabular}{|c|c|c|c|c|c|c|c|c|}
\hline Variabels & Source & $\begin{array}{l}\text { Type III Sum of } \\
\text { Squares }\end{array}$ & df & Mean Square & $\mathbf{F}$ & Sig. & $\begin{array}{l}\text { Partial Eta } \\
\text { Squared }\end{array}$ & $\begin{array}{c}\text { Observed } \\
\text { Powerb }\end{array}$ \\
\hline \multirow{3}{*}{$\begin{array}{l}\text { Avoidance of } \\
\text { death }\end{array}$} & Group & 18.971 & 1 & 18.971 & & & & \\
\hline & Error & 41.367 & 23 & 1.799 & 10.548 & 0.004 & 0.314 & 0.875 \\
\hline & Total & 531.920 & 26 & & & & & \\
\hline \multirow{3}{*}{ Quality of life } & Group & 31.669 & 1 & 31.669 & & & & \\
\hline & Error & 3054.567 & 23 & 132.807 & 0.238 & 0.630 & 0.010 & 0.075 \\
\hline & Total & 95468.750 & 26 & & & & & \\
\hline \multirow{3}{*}{ Mental health } & Group & 666.054 & 1 & 666.054 & & & & \\
\hline & Error & 1358.577 & 23 & 59.069 & 11.276 & 0.003 & 0.329 & 0.895 \\
\hline & Total & 82465.278 & 26 & & & & & \\
\hline \multirow{3}{*}{ Fear of death } & Group & 2.585 & 1 & 2.585 & & & & \\
\hline & Error & 8.090 & 23 & 0.352 & 7.350 & 0.012 & 0.242 & 0.738 \\
\hline & Total & 413.367 & 26 & & & & & \\
\hline \multirow{3}{*}{$\begin{array}{l}\text { Accept and } \\
\text { action }\end{array}$} & Group & .033 & 1 & 0.033 & & & & \\
\hline & Error & 23.104 & 23 & 1.005 & 0.033 & 0.858 & 0.001 & 0.053 \\
\hline & Total & 375.602 & 26 & & & & & \\
\hline
\end{tabular}


not in other dimensions of quality of life and psychological flexibility variable $(\mathrm{P}>0.001)$ at pretest, posttest, and followup stages (Table 1). In overall, the ACT significantly increased the mental health $(\mathrm{P}<0.001)$ and decreased the death avoidance and fear of death $(\mathrm{P}<0.001)$. It could $32.9 \%$ of variance in mental health, $20.9 \%$ in avoidance of death, and $28 \%$ in fear of death was due to the ACT-based intervention.

\section{Discussion}

ACT-based intervention could significantly decrease the fear of death and avoidance of death and improve the mental health of cancer patients. The main therapeutic goal of ACT is to decrease avoidance behaviors and increase the tendency towards experiencing disturbing feelings and thoughts. The results showed that the designed ACT package can meet this therapeutic goal.

\section{Ethical Considerations}

\section{Compliance with ethical guidelines}

This study obtained its ethical approval from the University of Isfahan (Code: IR.UI.REC.1396.047) and was conducted in cooperation with the Cancer Prevention Research Center of Isfahan University of Medical Sciences. Participants were assured of the confidentiality of their information. The purpose of study was explained to them and signed a written consent form. They were free to leave the study at anytime.

\section{Funding}

This research was extracted from the $\mathrm{PhD}$. thesis of second author approved by the Department of Education and Psychology, University of Isfahan in Isfahan, Iran.

\section{Authors contributions}

Conceptualization: Mohammad Bagher Kajbaf \& Seyed Ali Kolahdouzan; Methodology: Mohammad Bagher Kajbaf; validation, data analysis, editing, project administration: All authors; Investigation \& resources: Seyed Ali Kolahdouzan; Draft preparation, writing \& review: Seyed Ali Kolahdouzan.

\section{Conflicts of interest}

The author declared no conflict of interest

\section{Acknowledgements}

The authors would like to thank Cancer Prevention Research Center of Isfahan's Seyedoshohada Hospital for their contribution in this research. 


\title{
تدوين و بروسى اثربخشى بسته درمانى (اضطراب از مركَ)) مبتنى بر درمان يذيرش و تعهيد بر

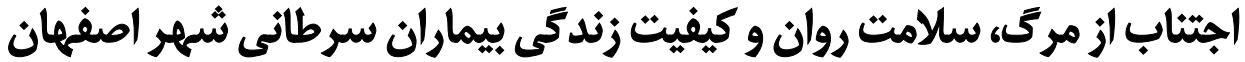

سيد على كلاهدوزان' •، •محمدباقر كجباف' •، حميد رضا عريضى'، محمد رضا عابدى'، فريبرز مكاريان'

ا. ا.كروه روانشناسى، دانشكده علوم تربيتى و روانشناسى، دانشكاه اصفهان، اصفهان، ايران.

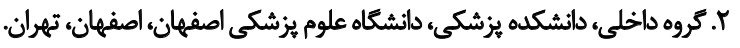

\begin{abstract}
حكيد

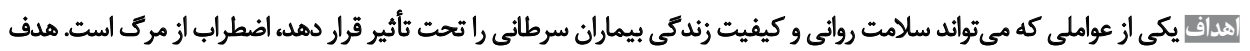

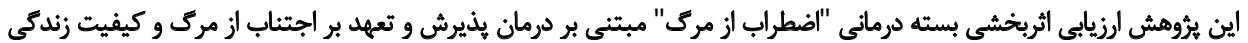
بيمار ان سرطاني مى باشد الشري

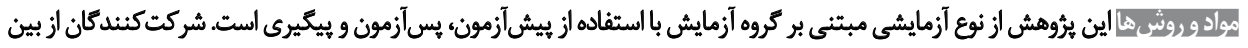

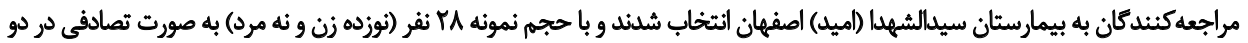

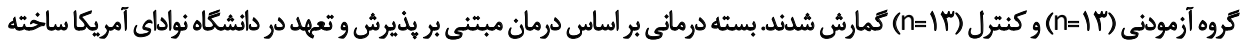

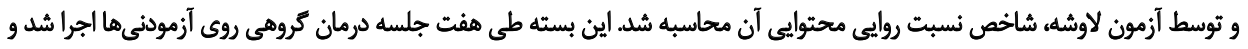

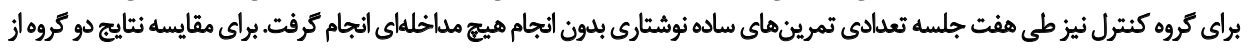

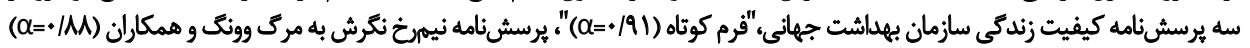

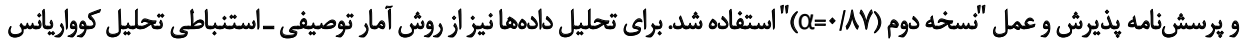

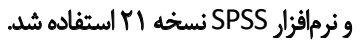

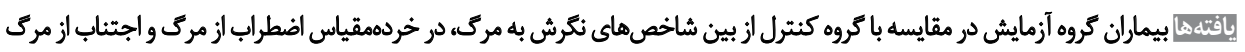

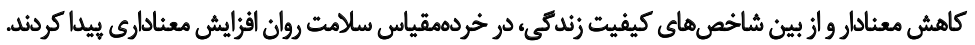

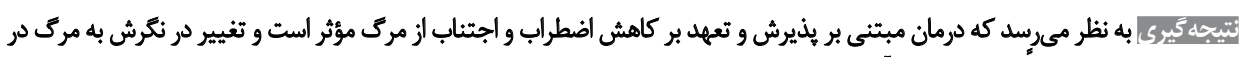
بيماران سرطانى احتمالأبا سلامت روان آنها مرتبط رست است.
\end{abstract}

تاريخ دريافت: ال شهريور IFA

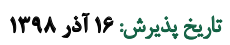

تاريخ انتشار: rا فروردين

كليدواثهها:

درمان مبتنى بر بذيرش

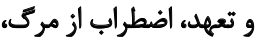

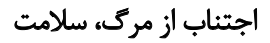

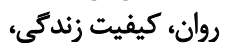
سرطان

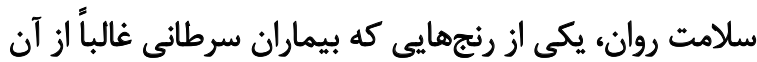
dale

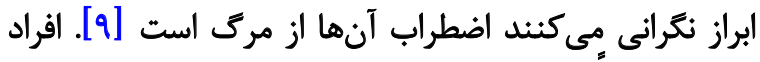

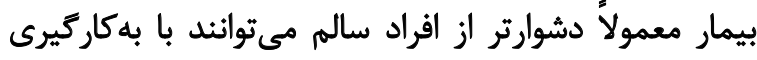

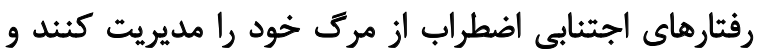

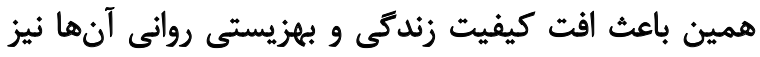

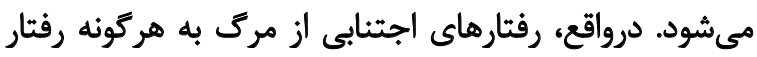

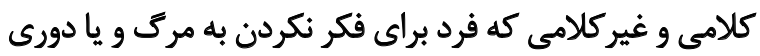

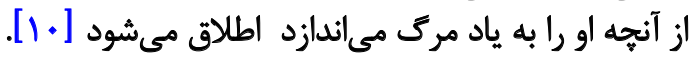

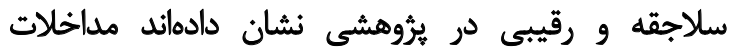

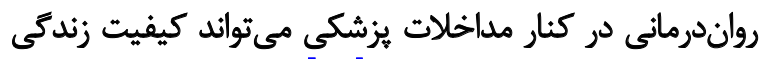

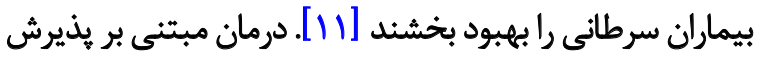

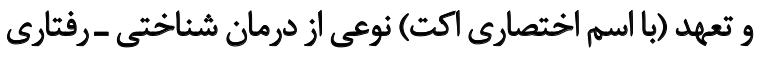

طبق گَزارش سازمان بينالمللى تحقيقات سرطان' (1) - (Y)،

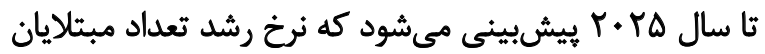

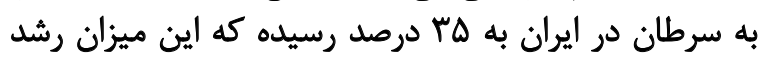

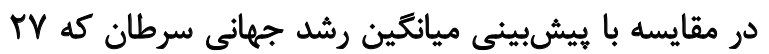

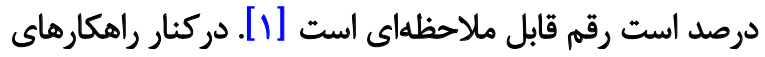

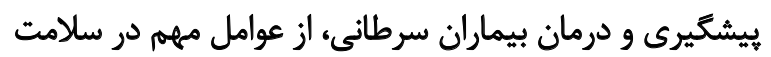

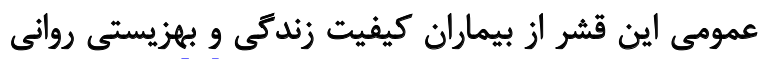

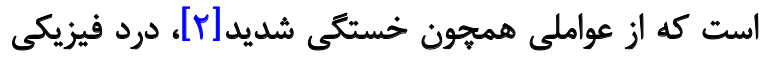

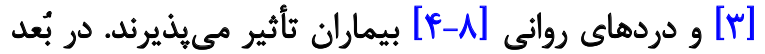

1. International Agency for Research on Cancer (IARC)

$$
\text { - }
$$

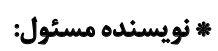

دكتر محمدياقر كجباف

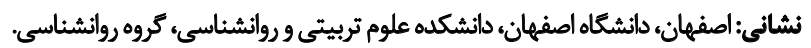
تلفن: m.b.kaj@edu.ui.ac.ir يست الكترونيكي 


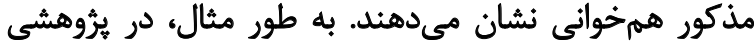

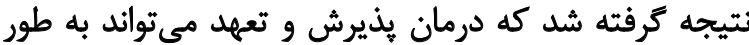

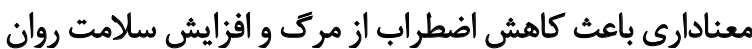

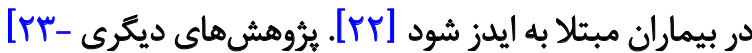

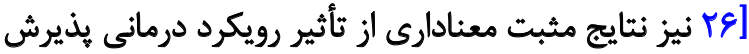

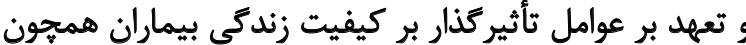

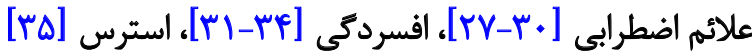
و درد ناشى از اضطراب [عب] كزارش دادهانداند.

به طور معمول در درمان اضطراب از مرك، رواندرمانتران

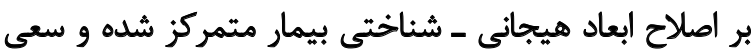

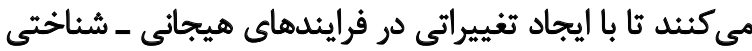

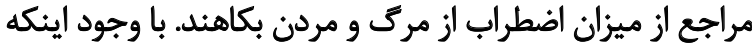

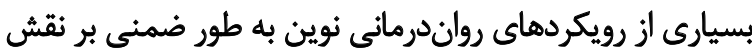

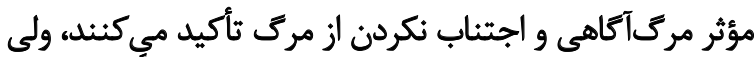

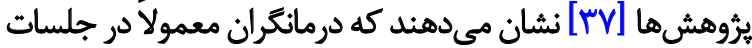

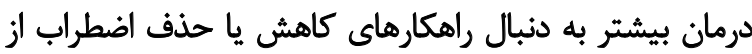

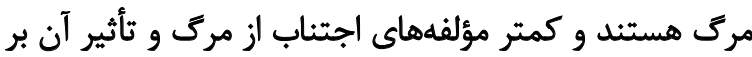

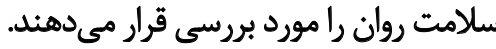

اجتناب از مرك به هركونه رفتار كلامى و غير كلامى كه فرد

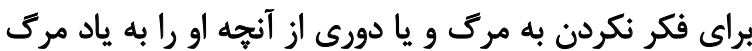

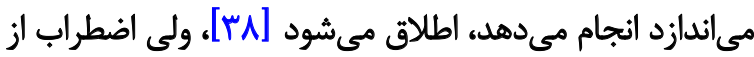

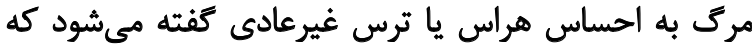

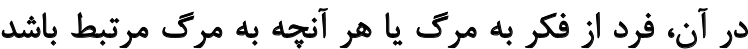

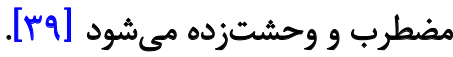

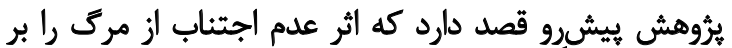

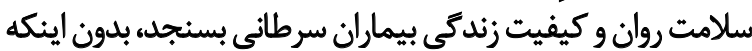

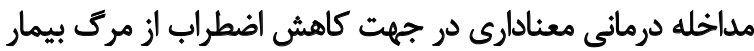

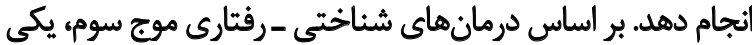

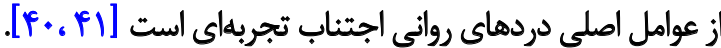

يافتهايى تحقيقات روى بيماران مبتلا به سرطان سينه نشان

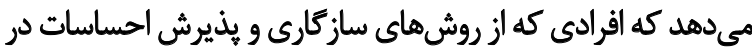

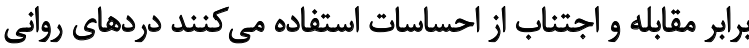

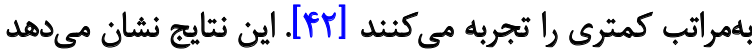

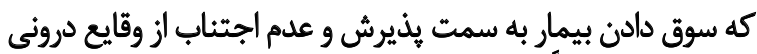

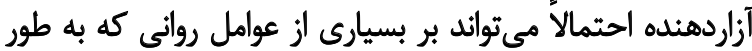
مستقيم نقش تعيين كنتده در سلامت روان بيماران دارئ بارد، تأثير كذارد.

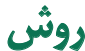

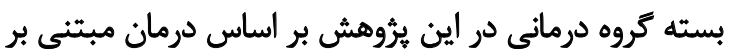

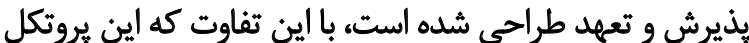

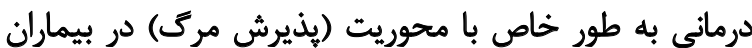

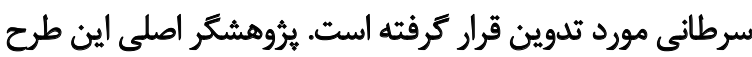

است كه مبتنى بر زمينهكرايى عملكردى جار و بر اساس نظريه

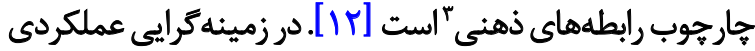

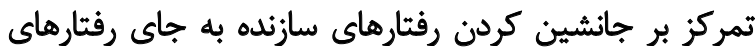

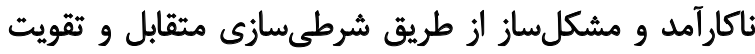

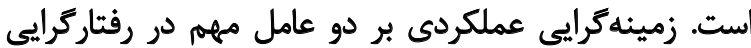

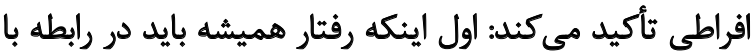

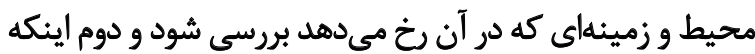

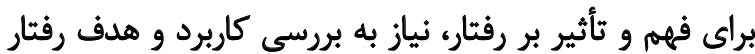

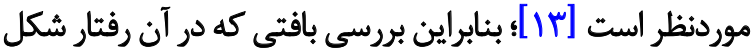

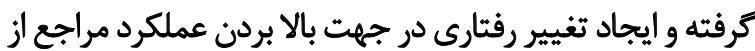

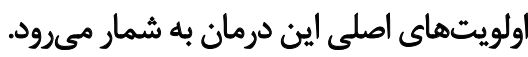

درمان مبتنى بر بذيرش و تعهد هميجون ساير درمانهاي

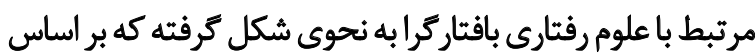

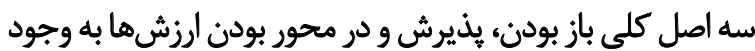
آمده است و هدف اصلي درماني اين رويكرد ايجاد انعطافئيديرى دئي

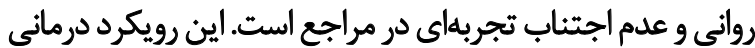

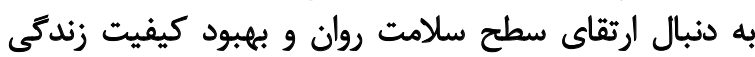

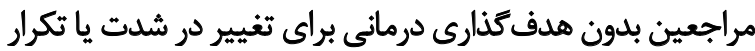

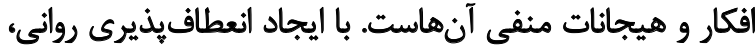

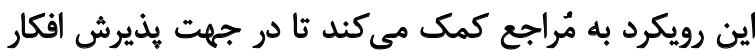

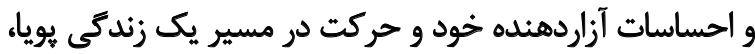

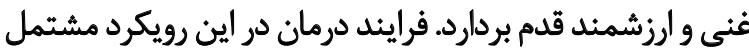

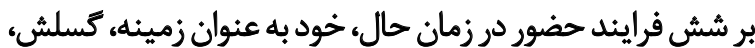

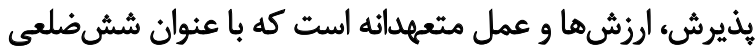

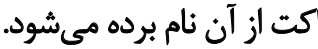

بسيارى از بيمارانى كه با سرطانهاى بيشرفته زندكى مي كنئن

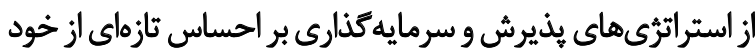

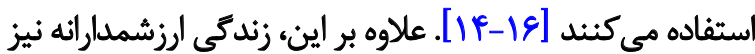

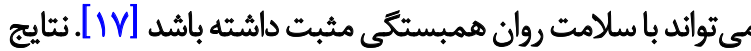

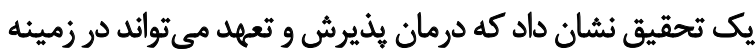

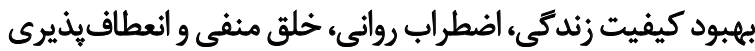

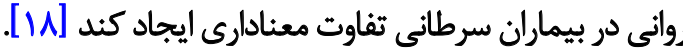
درمان بذيرش و تعهيد همجنين به افراد كمك مى إكند

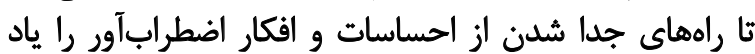

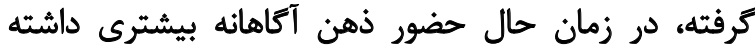

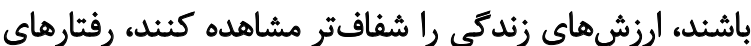

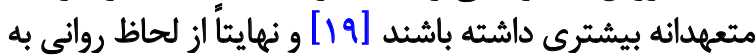

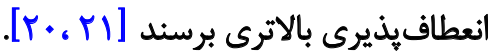
در ايران نيز بيروهشهايى براي ارزيابى تأثير رويكرد درمانى باني

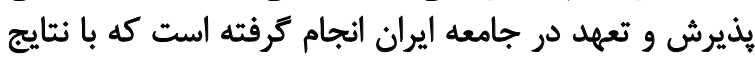

2. Functional contextualism

3. Relational frame theory (RFT) 
شركت كنندكان در تمامى جلسات به صورت حضورى شركت

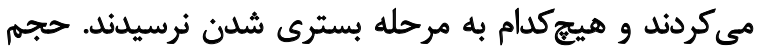

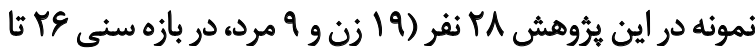

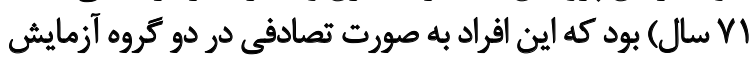

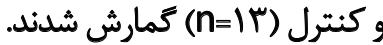

از بر نفر شركتكنيده، دو نفر به دليل بعد مسافت و و

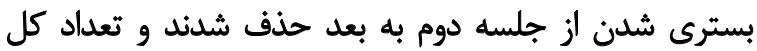

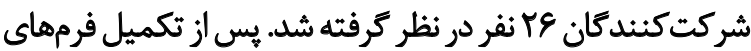

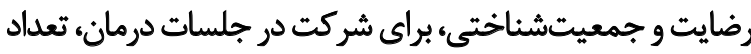

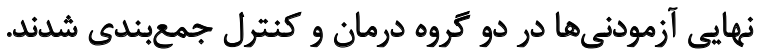

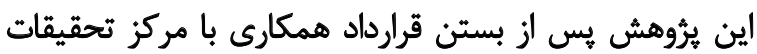

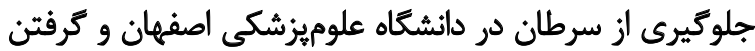

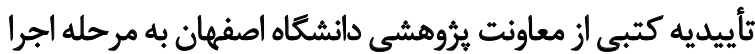

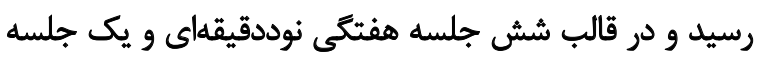

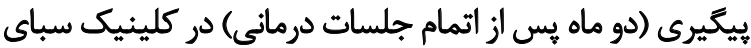
اصفهان انجام كرفت (جدول شماره () اتمات جات دماني

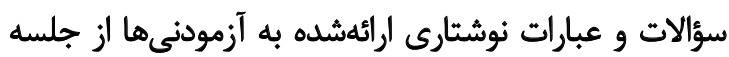

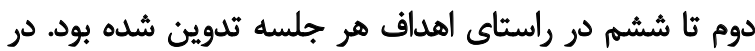

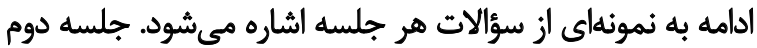

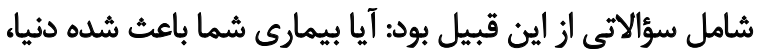

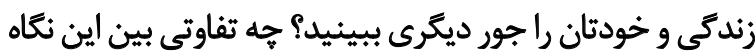

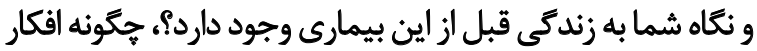

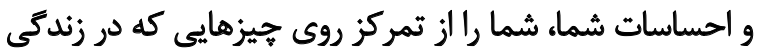

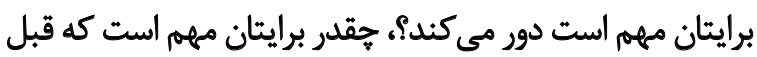

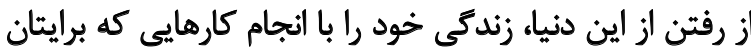

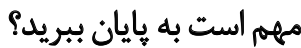

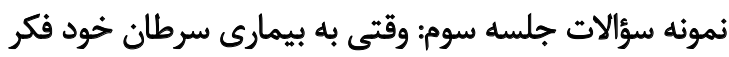

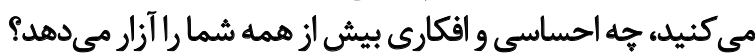

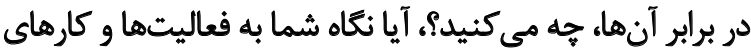

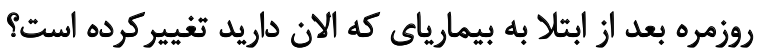

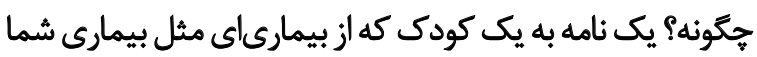

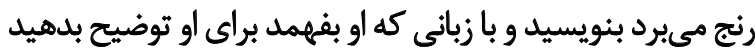
كه جطور مى تواند با بيمارى اش زندئ زئى كند.

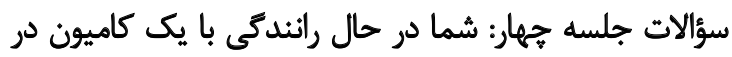

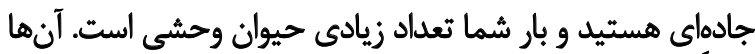

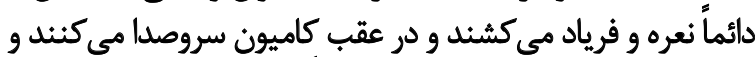

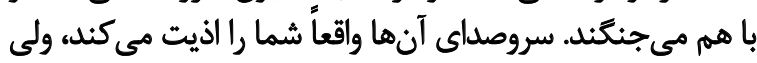

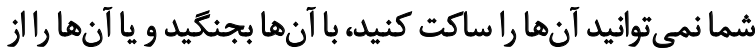

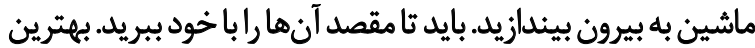

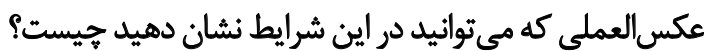

در جلسه ينجمه، سؤالاتى از اين قبيل با بيمار مطرح شد: تصور كنيد

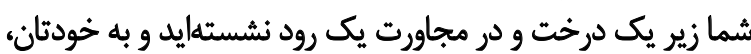

در طي يك دوره فرصت مطالعاتى دماهاه در دانشكاه نواداي

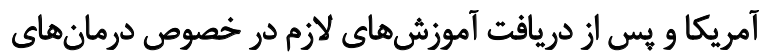

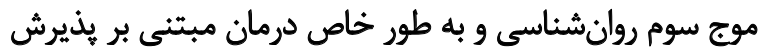

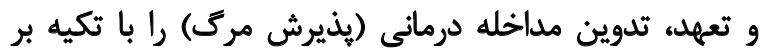

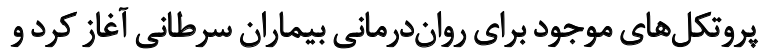

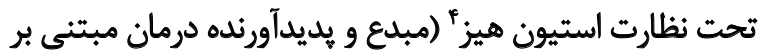

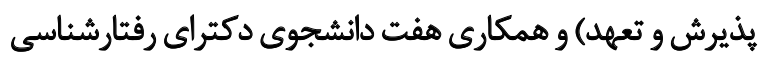

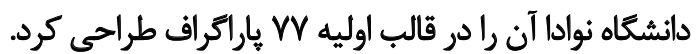
در مرحله بعد جهت گَترش محتواى بسته درمانى،

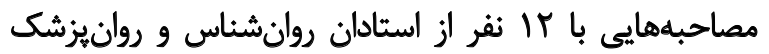

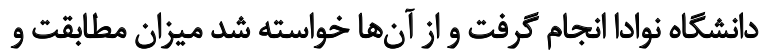

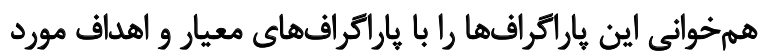

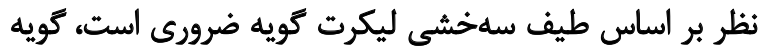

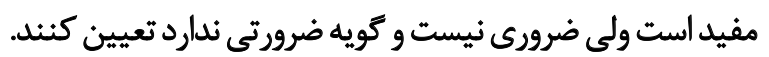

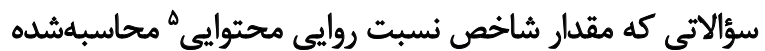

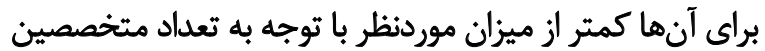

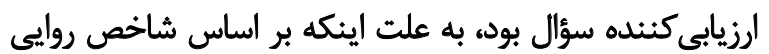

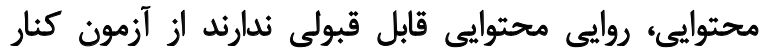

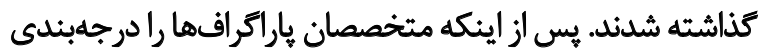

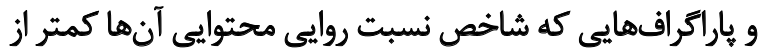

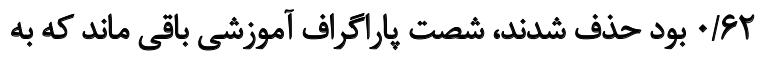
ياراترافهاى اصلى اضافه شدند.

تدوين اين بسته تحت نظارت يروفسور استيون هيز و و

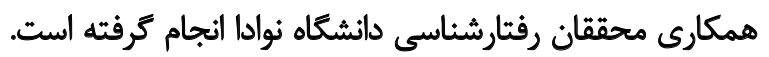

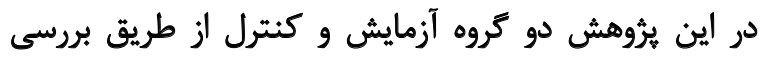

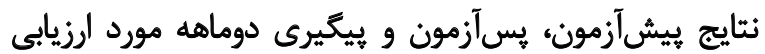

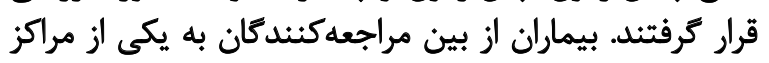

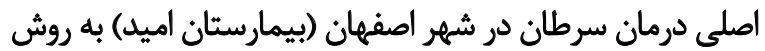

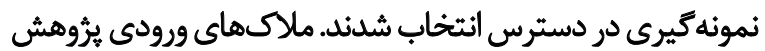

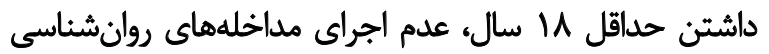

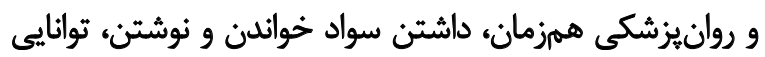

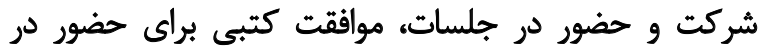

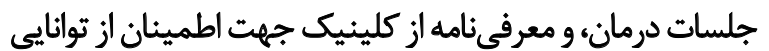
جسمى فرد براى حضور در جلسات بودمان

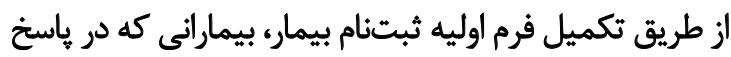

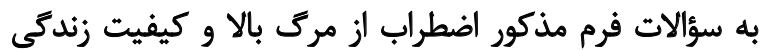

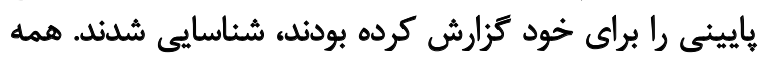

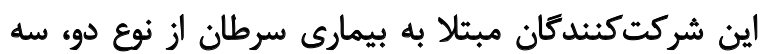

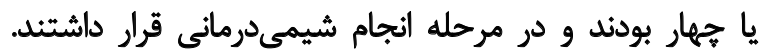

4. Steven C. Hayes; Nevada Foundation Professor at the University of Nevada, Reno Department of Psychology 5. CVR 
جدول 1. اهداف و روش اجراى جلسات درمانى

\begin{tabular}{|c|c|c|}
\hline اجرا & هدف & جلسه \\
\hline ايجاد انعطافيذيرى روائى با وجود افكار و احساسات آزاردهنده & انعطافيذيرى روائي نسبت به مرى و مردن & بسته درماني \\
\hline 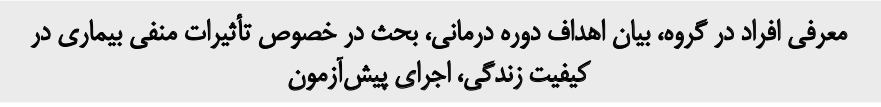 & معارفه & جلسه اول \\
\hline 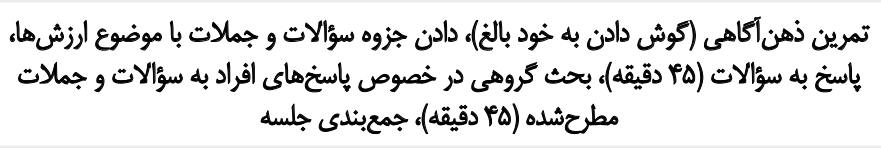 & حركت به سمت ارزشهاي فردى & جلسه دومج \\
\hline 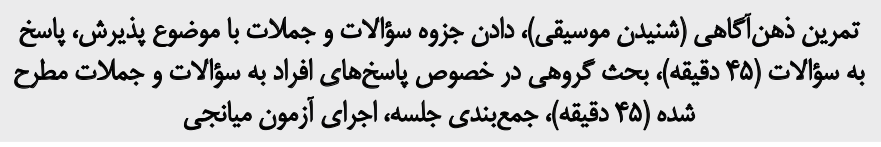 & عبور از اجتناب تجربهاى و حركت به سمت & جلسه سوم \\
\hline 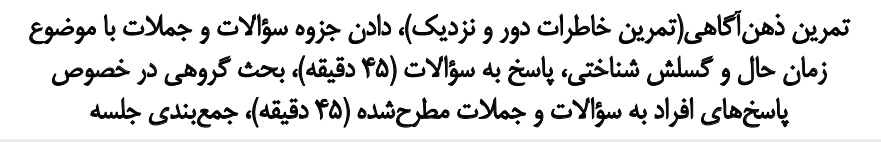 & 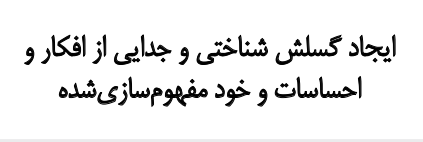 & جلسه جهارم \\
\hline 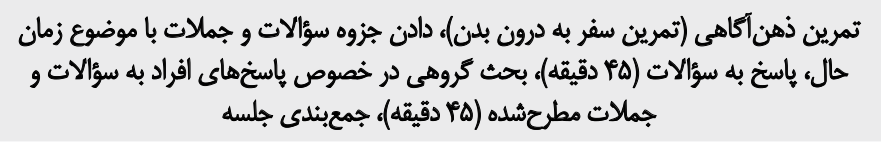 & ارتباط با زمان حال و آششنائي با كنشته و & جلسه هنجم \\
\hline 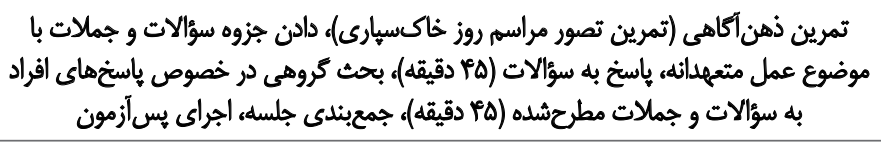 & انجام عمل متعهدانه در راستاى ارزش هاى & جلسه ششم \\
\hline
\end{tabular}

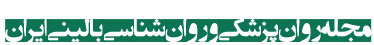

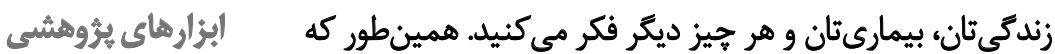
سه برسشنامه براى ارزيابى اين ثئوهش در نظر كرفته

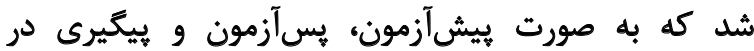

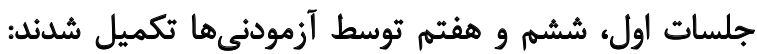

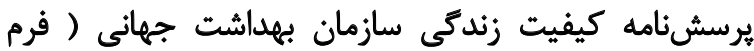

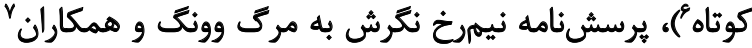

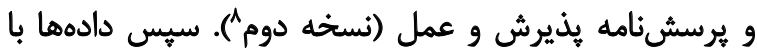

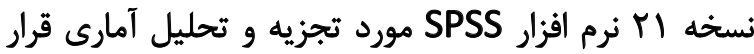

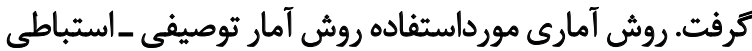

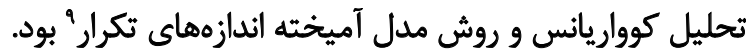

$$
\text { كيفيت زندكّ و سلامت ووان }
$$

اين دو عامل توسط يرسش نامه كيفيت زندكى سازمان بهداشت

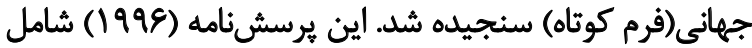

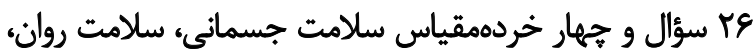

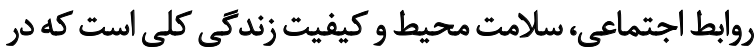

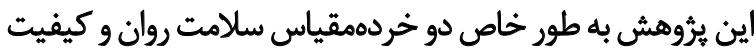

6. WHOQOL-BREF

7. DAP-R

8. AAQ-II

9. Mixed model repeated measures (MMRM)

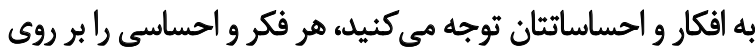

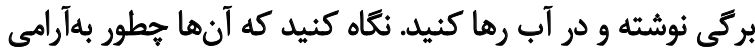

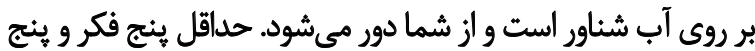

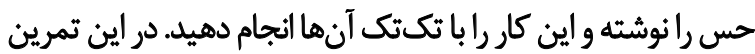

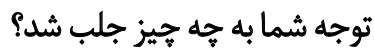

بلموازات كروه آزمايش براى كروه كنترل نيز به همين تعداد

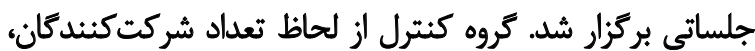

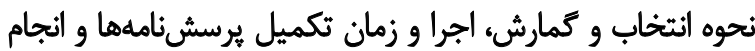

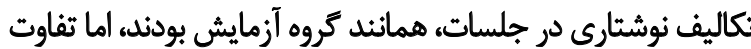

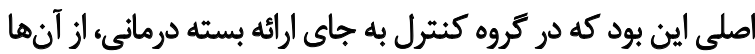

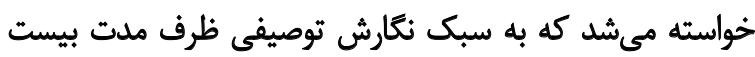

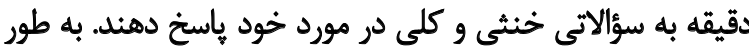

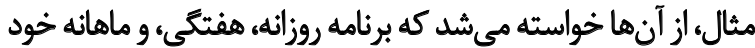

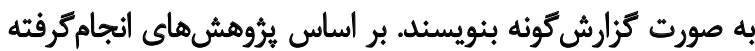

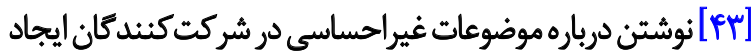
تغيير معنادارى نمى كند.

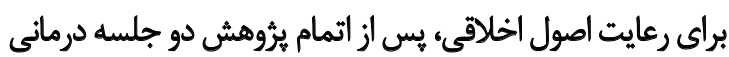

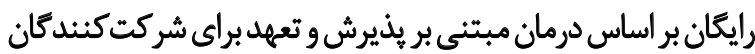
كذاشته شد كه رضايت نسبى آنها را به همراه دان داشت 


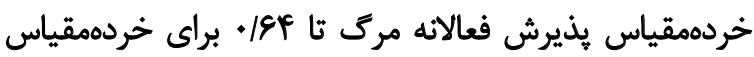

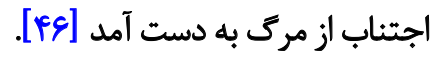

\section{انعطافئذيرى روانى}

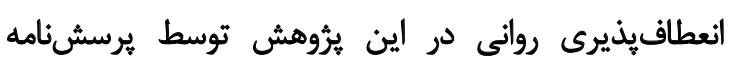

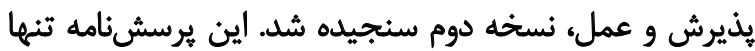

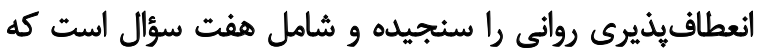

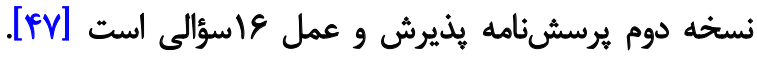

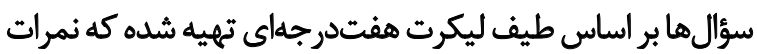

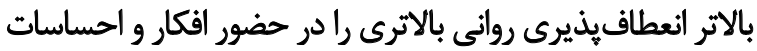

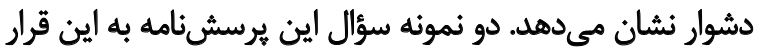

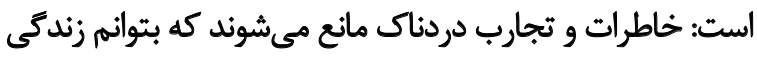

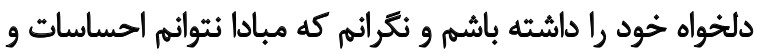

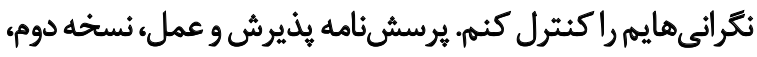

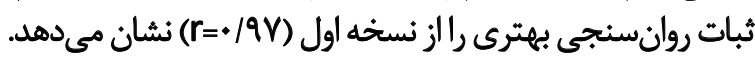

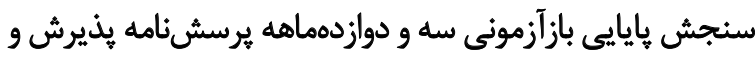

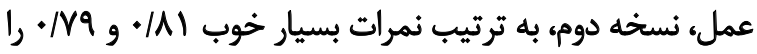

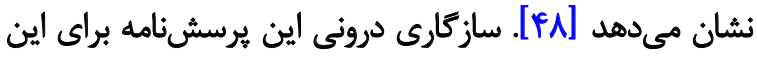

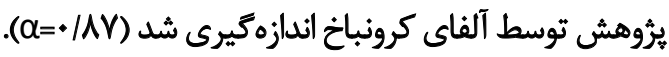

يافتهها

براى مقايسه آزمودنىهاى هر كروه آزمايشى، اطلاعات

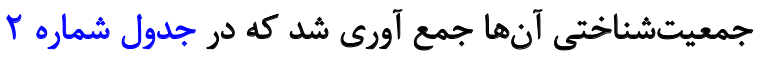

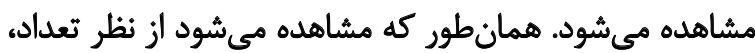

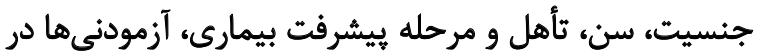

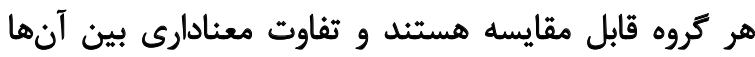

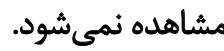

با توجه به اينكه ياسخ كويى به سؤالات اصلى يُورهش باديا

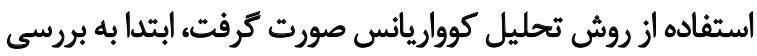

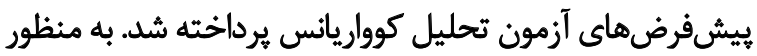

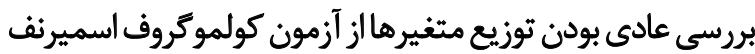

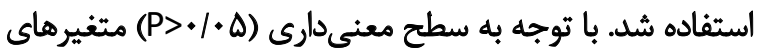

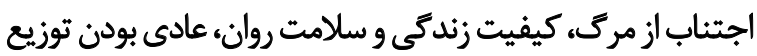

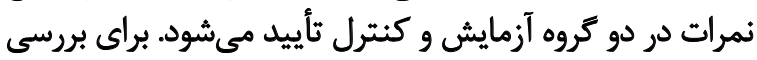

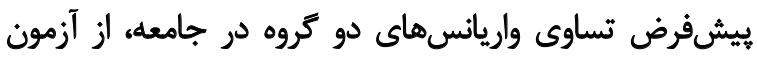

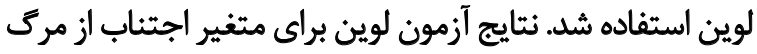

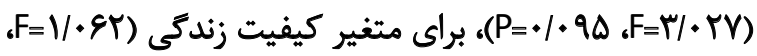

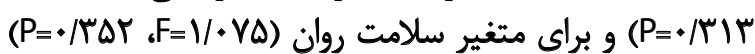
نشانتر تحقق ييشفرض تساوى واريانس ها بودي

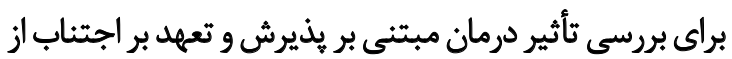

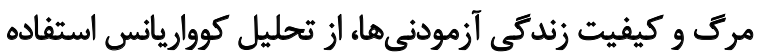

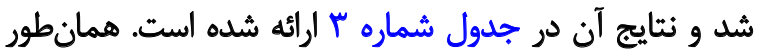

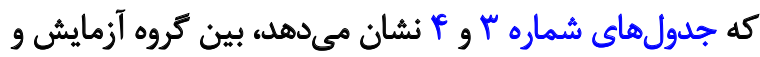

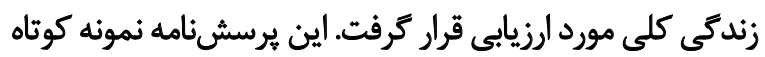

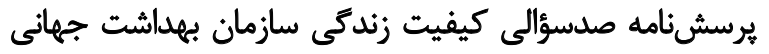

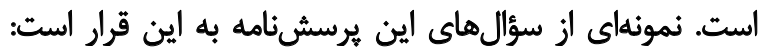

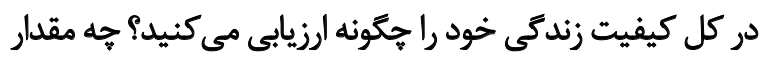

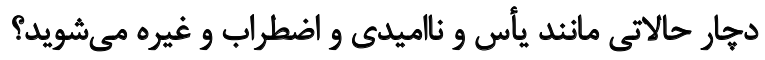

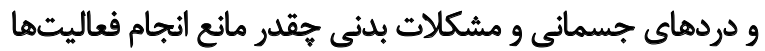

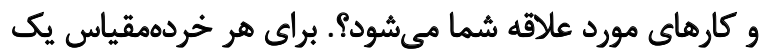

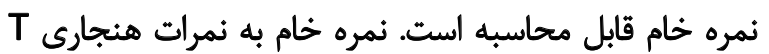

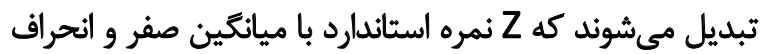

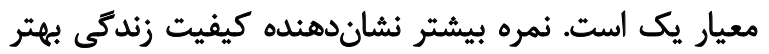

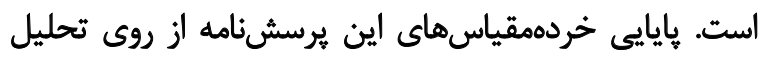

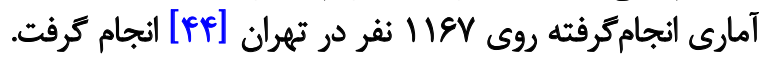

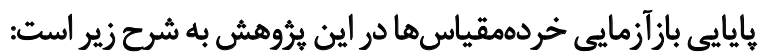

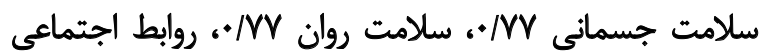

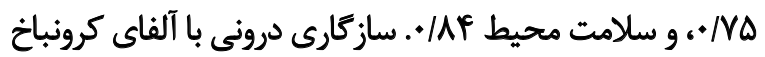

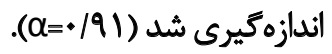

\section{اضطراب أز مرتى و اجتناب أز مركى}

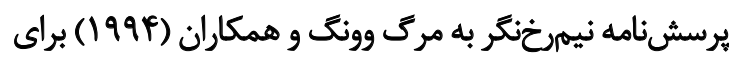

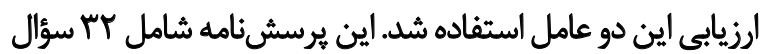

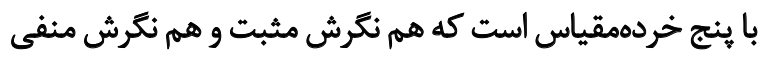

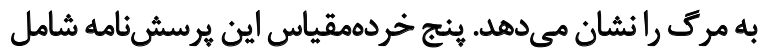

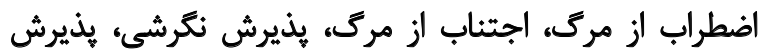

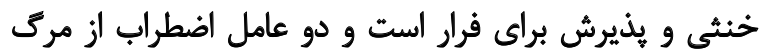

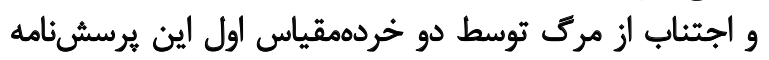

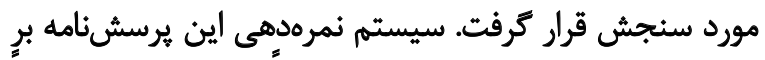

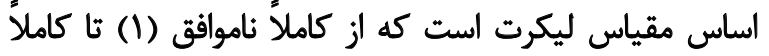

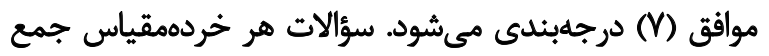

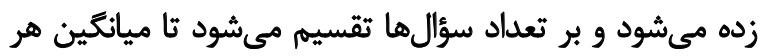

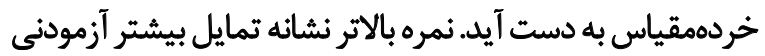

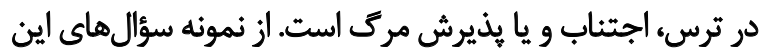

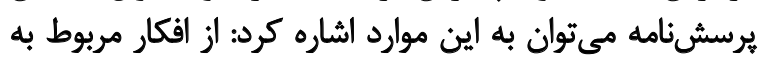

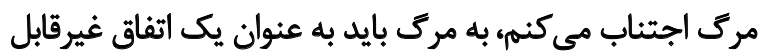

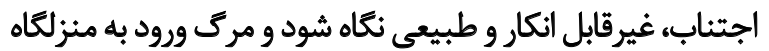

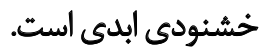

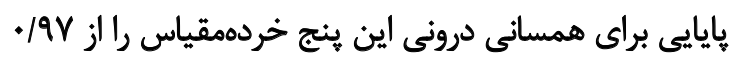

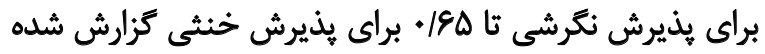

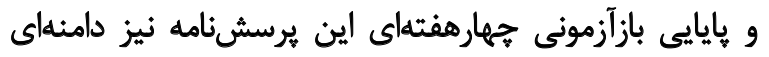

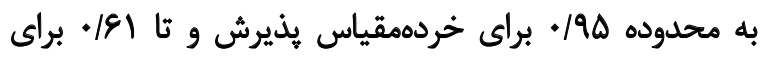

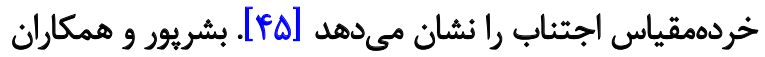

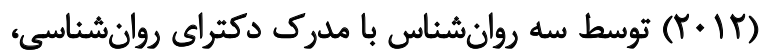

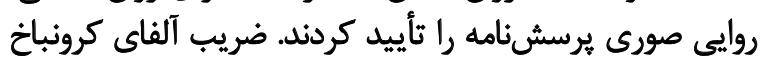

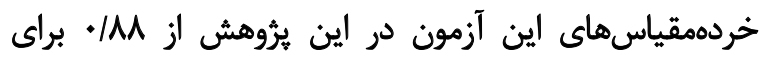


جدول r. اطلاعات جمعيتشناختى شركت كند كان در يثوهش

\begin{tabular}{|c|c|c|c|c|c|c|c|}
\hline \multirow{2}{*}{ مرحله سرطان (ا ابتدايى - ع عيشئه } & \multicolumn{2}{|c|}{ ت تأهل } & \multirow[t]{2}{*}{ ميانكين سن } & \multicolumn{2}{|c|}{ جنسيت } & \multirow{2}{*}{ شر كت كثندادكان } & \multirow[t]{2}{*}{ اطلاعات جمعيتشناختى } \\
\hline & مثأهل & 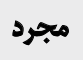 & & مرد & ز & & \\
\hline$r / \Lambda \Delta$ & 1. & $\varphi$ & $P T / M$ & f & 1. & if & كروه درمان مبتنى بر يذيرش \\
\hline \multirow[t]{2}{*}{$T / W$} & 11 & $r$ & PAIAT & $\Delta$ & 9 & if & كروه كتترل \\
\hline & $p q$ & r & & 9 & 19 & rA & كل كل \\
\hline
\end{tabular}

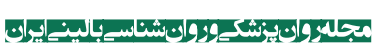

جدول س. نتايج تحليل كوواريانس براى بررسى ثأثير درمان مبتنى بر هذيرش و تعهد بر اجتناب از مركى و كيفيت زندكى آزمودئىها

\begin{tabular}{|c|c|c|c|c|c|c|}
\hline سطح محنى دارى & توان آمارى & درجه آزادى خطا & درجه آزادى فرضيه & $\mathbf{F}$ & إزڤ & آزمون \\
\hline$P<+1.0$ & . / a & 19 & f & $\mathrm{V} / \mathrm{WW}$ & $\cdot 18+1$ & اثر ييلاى \\
\hline$P<+1 \cdot \Delta$ & . /ur & 19 & f & $V / r W$ &.$/$ rar & لامبداى ويلكز \\
\hline$P<+1+\Delta$ & tarr & 19 & f & $V / r W$ & V/AQT & اثرهتلينى \\
\hline$P<+1 . \Delta$ & . /ur & 19 & $r$ & $\mathrm{~V} / \mathrm{m}$ & V/DAT & بزركترين ريشه \\
\hline
\end{tabular}

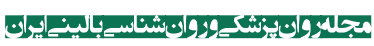

جدول f. ميانكين، انحراف معيار اجتناب از مرك، كيفيت زندكى و سلامت روان در مراحل مختلف

\begin{tabular}{|c|c|c|c|}
\hline ميانكين 土|نحراف معيار & كروه & مرحله & مثغير \\
\hline$f / q f \pm V / q 4$ & آزمايش & \multirow{2}{*}{ يبش آزمون } & \multirow{6}{*}{ اجتناب از مرك } \\
\hline$\Delta /+\Phi \pm+M^{\top}$ & كتنتل & & \\
\hline$r / M I \pm V / \& 8$ & آلزمايش & \multirow{4}{*}{ هيس آزمون } & \\
\hline$\Delta / M \pm / /$ & كثترل & & \\
\hline$r / r \Delta \pm V / \Delta q$ & أزئهايش & & \\
\hline$\Delta / m \Psi \pm V \cdot r$ & 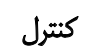 & & \\
\hline $8 \cdot / \Delta V \pm I D / I V$ & آزمايش & \multirow{2}{*}{ ييش آز } & \multirow{6}{*}{ كيفيت زندكى } \\
\hline $0 . / Q \& \pm T Y / \Delta)$ & 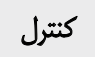 & & \\
\hline$g r / \Delta \cdot \pm 19 / \cdot q$ & آزازمايش & \multirow{4}{*}{ يسيكيرى ئزمون } & \\
\hline Q)/QY $\pm r \cdot / 9 \varphi$ & 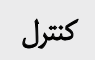 & & \\
\hline $98 / \pi \pm 1 \cdot / 9 A$ & آزمايش & & \\
\hline$r q / . r \pm 1 q / r$. & 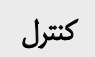 & & \\
\hline$\Phi / / \Lambda \Psi \pm \mid \Delta / \Psi^{\prime} \Delta$ & آزمايش & \multirow{2}{*}{ يبش آزمون } & \multirow{6}{*}{ سلامت روان } \\
\hline DS/NEITY/TY & كتترل & & \\
\hline SE/VDDID/SE & آزآزمايش & \multirow{2}{*}{ بس آزمون } & \\
\hline$\Delta E / T I \pm r / M^{*}$ & 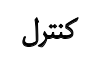 & & \\
\hline$E N / T \cdot \pm 1 \cdot / r q$ & آز آزهايش & \multirow{2}{*}{ ييكيرى } & \\
\hline$\Delta V / Q \& \pm Y V / Q A$ & كنترل & & \\
\hline
\end{tabular}


جدول هـ نتايج حاصل از تحليل آنكووا در متن مانكووا روى تفاضل ميانكين نمرههاى بيش آزمون و يس آزمون اجتناب از مركى، كيفيت زندكى و سلامت روان در

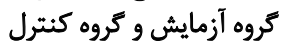

\begin{tabular}{|c|c|c|c|c|c|c|c|c|}
\hline معنى سطارى & توان آمارى & الندازه اثر & $\mathbf{F}$ & مجذئزين مئورات & درجه آزادى & مجموع مجذورات & منيع تغيير & متغير \\
\hline \multirow{3}{*}{$<+1+\infty$} & \multirow{3}{*}{.1840} & \multirow{3}{*}{$+/ r+9$} & \multirow{3}{*}{$\Delta / A) \backslash / \varepsilon+\Delta$} & จ/941 & 1 & $9 / 901$ & كروه & \multirow{3}{*}{ اجتناب از مرى } \\
\hline & & & & \multirow[t]{2}{*}{ WIr } & M & rWISN & خطا & \\
\hline & & & & & re & DrVIar. & كل & \\
\hline \multirow{3}{*}{$>+1+\Delta$} & \multirow{3}{*}{.$/ 119$} & \multirow{3}{*}{. $r A$} & \multirow{3}{*}{. } & AT/ITH & 1 & AT/NRT & كروه & \multirow{3}{*}{ كيفيت زندكى } \\
\hline & & & & \multirow[t]{2}{*}{$1 r q / 7 \cdot 9$} & rr & TAFT/Q QA & خطا & \\
\hline & & & & & rq & QAFENVA. & كل - ال & \\
\hline \multirow{3}{*}{$<+1 \cdot \Delta$} & \multirow{3}{*}{. 1199} & \multirow{3}{*}{.$/ M T q$} & \multirow{3}{*}{ IV/TVE } & $8 g 8 / . \Delta f$ & 1 & $\operatorname{seg} \cdot \Delta f^{\circ}$ & كروه & \multirow{3}{*}{ سلامت روان } \\
\hline & & & & \multirow[t]{2}{*}{$\$ 91.89$} & r & ITENOW & خطا & \\
\hline & & & & & re & ATred/TVA & كل & \\
\hline
\end{tabular}

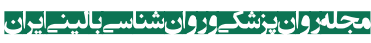

به شمار ميرود و مههمترين عامل مركى مير بعد از بيمارىهاى

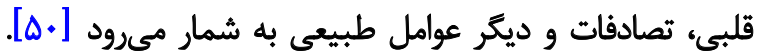

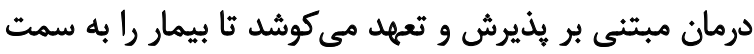

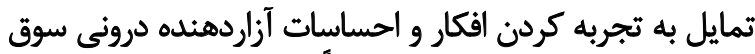

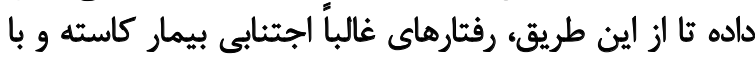

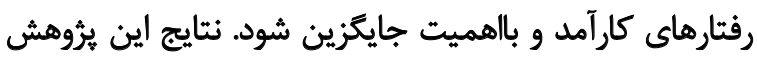

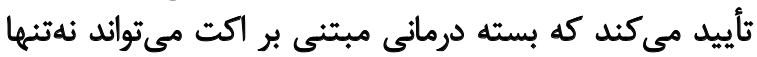

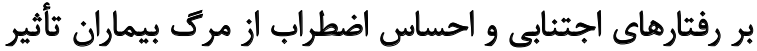

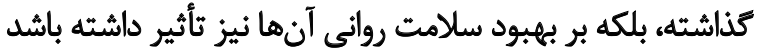

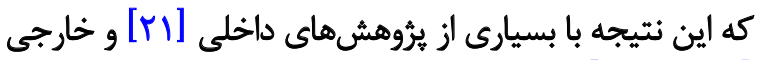

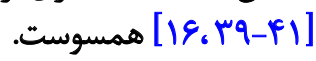

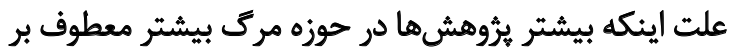

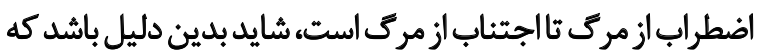

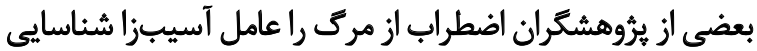

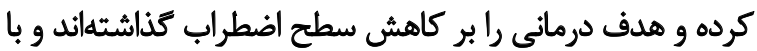

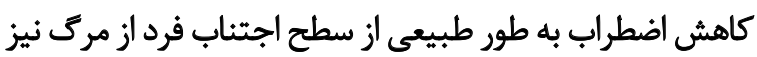

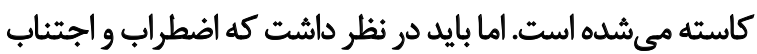

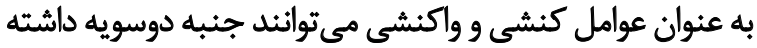

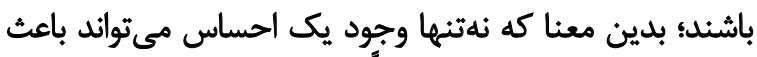

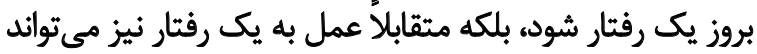

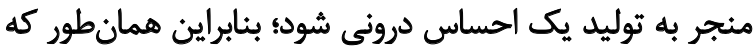

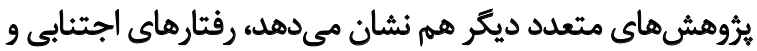

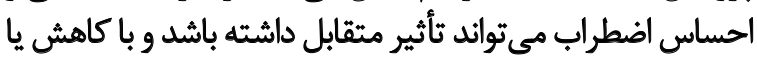

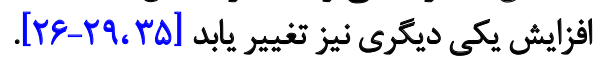

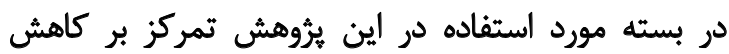

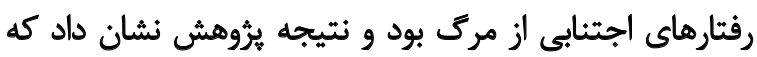

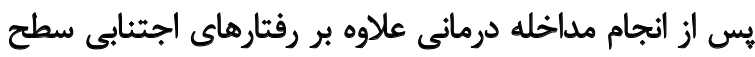

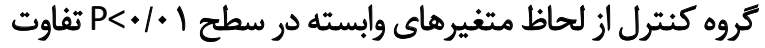

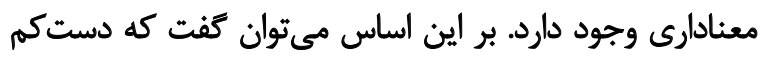

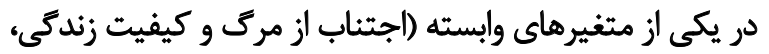

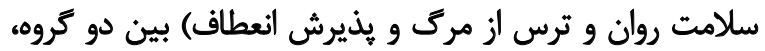

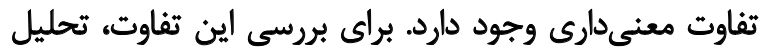

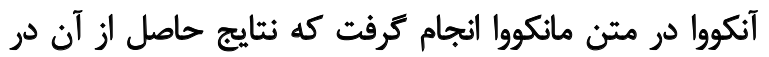
جدول شماره ه درج شده است. با توجه به ميانكينهاي ارائهشده و با توجه به سطح معنى دارى

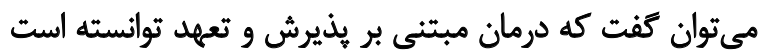

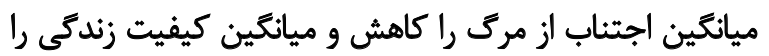

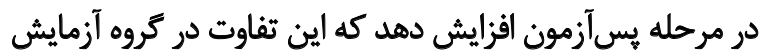

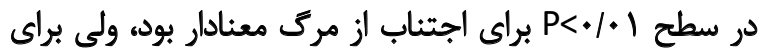

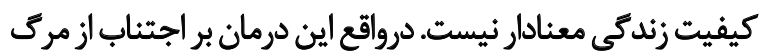

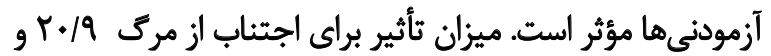

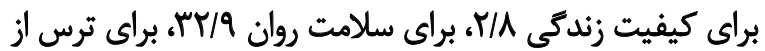

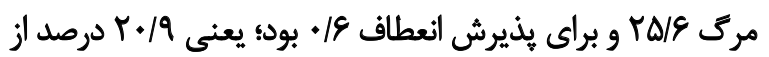

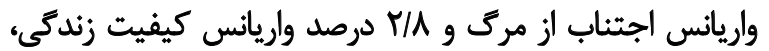

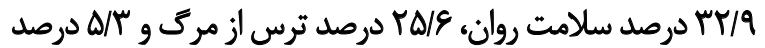

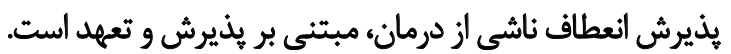

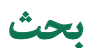

ايران به عنوان كشورى در حال توسعه كه در سالهاى اخير از

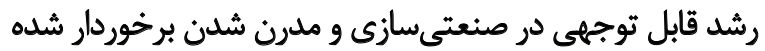

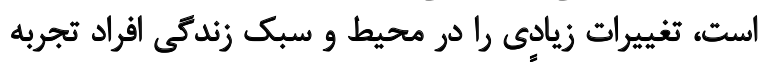

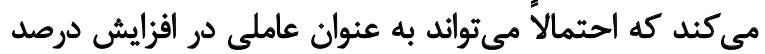

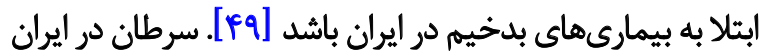
به عنوان دومين كروه بزرى از بيمارى برانهاى وخيم غيرواكيردار 
معنادارى دارند. اين نتايج تأييدكنثند اين قسمت ازئن نظريه درمان

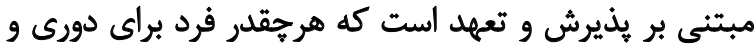

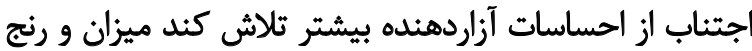

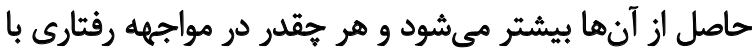

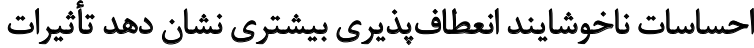
منفى و آسيبزاى كمترى را تجربه خواهد كرد. در خصوص ارتباط كيفيت زندكى بيماران و اجتناب تجربهاى

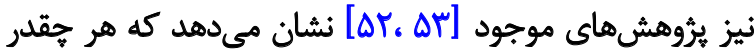

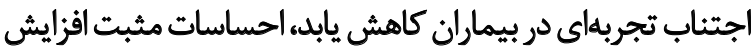

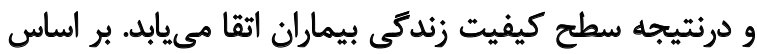

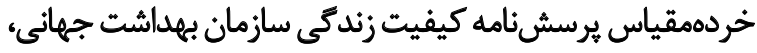

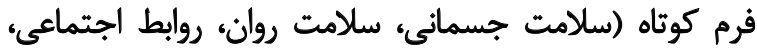

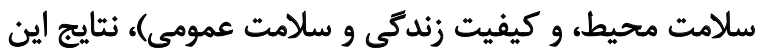
يرؤهش رامىتوان اين كونه تفسير كرد: كيفيت زندكى بيمار سرطانى تحت تأثير عوامل زيادى از جمله

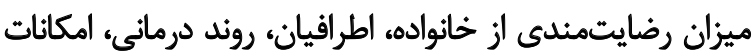

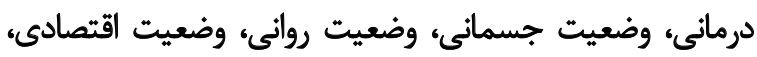

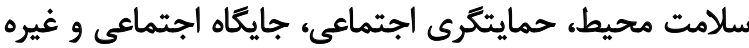

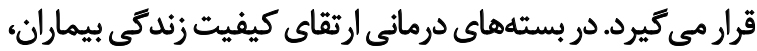

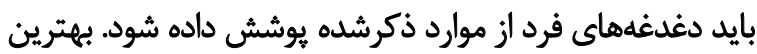

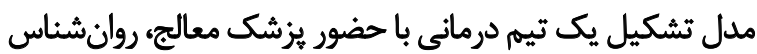

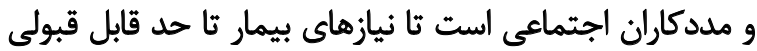

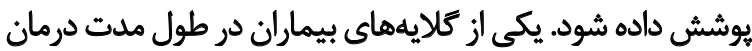

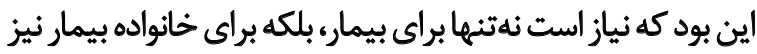

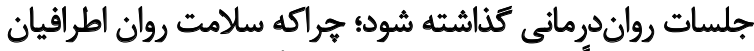
بيمار مستقيماً روى سلامت روان بيمار تأثير مى خذاردا.

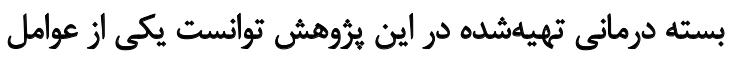

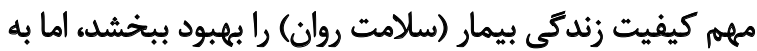

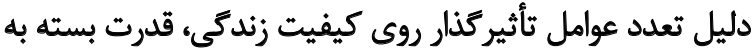

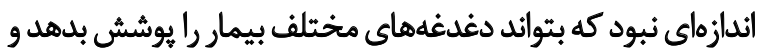

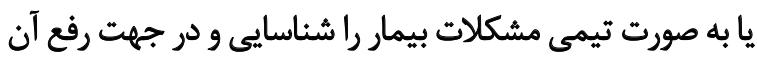

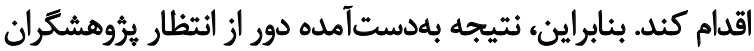

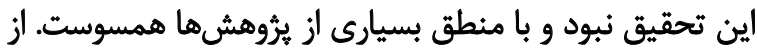

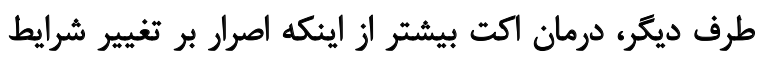

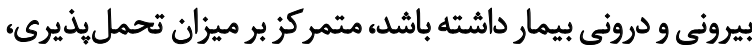

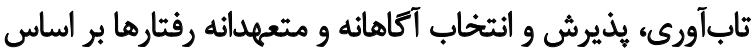

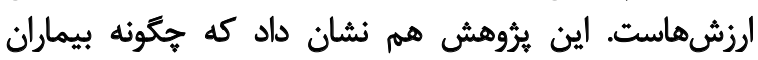

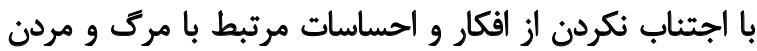

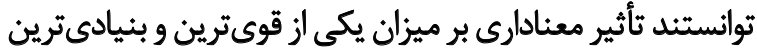

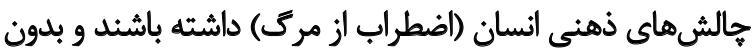

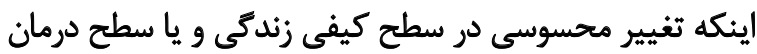
فيزيكى ايجاد شود، به سلامت روانى بالاترى دست بيديدا كنيند.

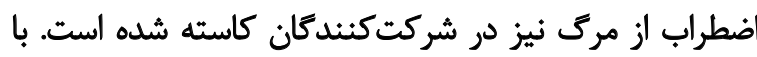

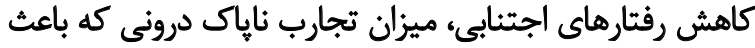

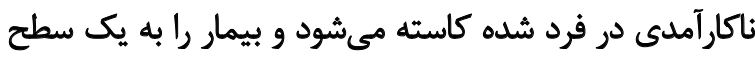

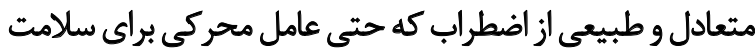

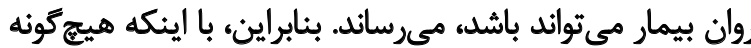

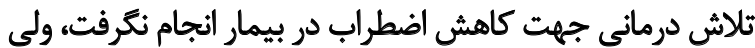

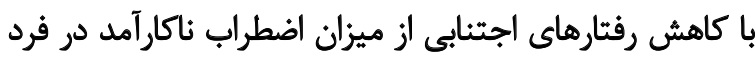

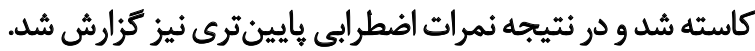

با وجود اينكه از ميزان اضطراب و اجتناب از مرك در بيماران

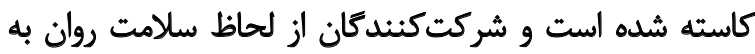

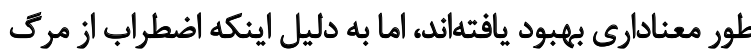

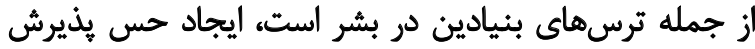

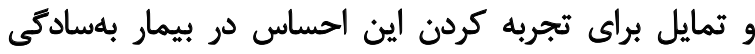

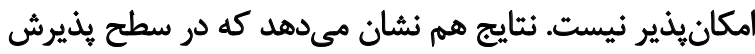

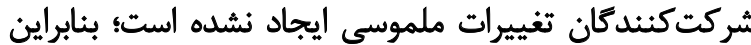

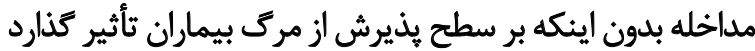

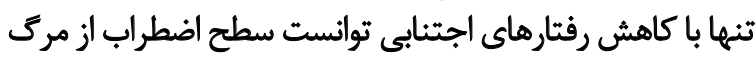

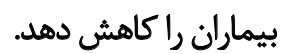

طبق نظريه اكت، يذيرش از اركان اصلى ايجاد انعطافيذيرى

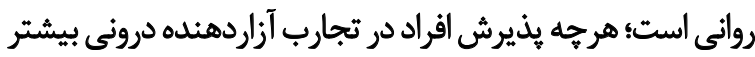

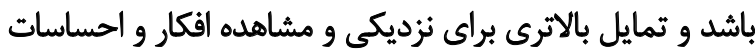

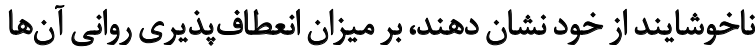

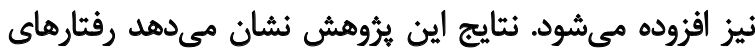

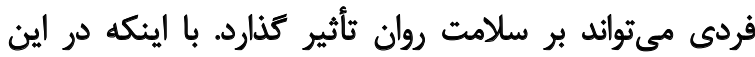

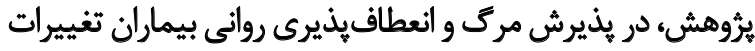

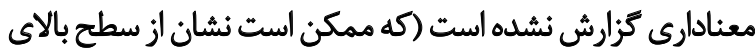

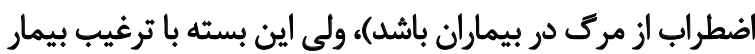

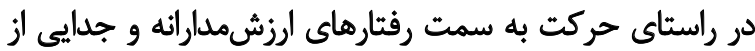

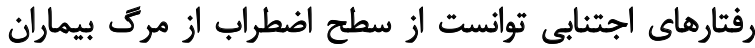

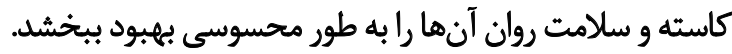
در بسيارى از كزارشات شفاهى كرفتهشده از شركت كنيندكان

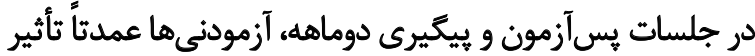

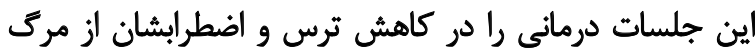

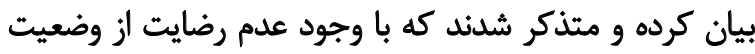

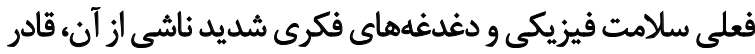

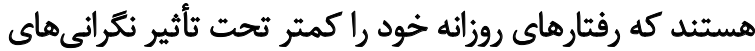

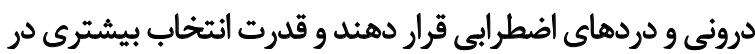

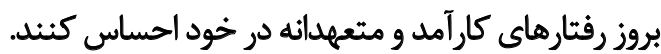

يُروهشهاي مشابه نيز نشان دادهاند كه عدم اجتناب در بيمار

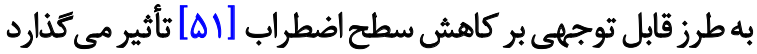

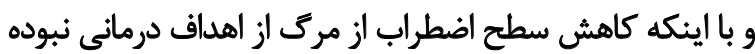

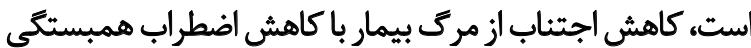




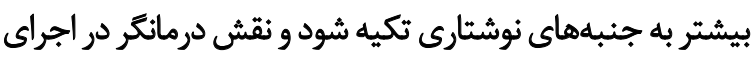

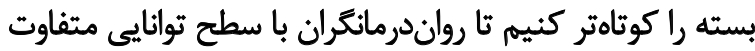

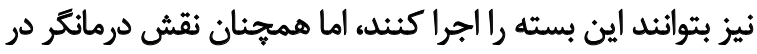

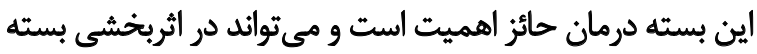

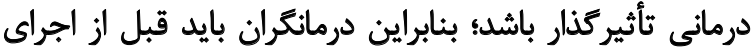
بسته، آموزش تانير لازمنار باشك راديده باين داشند.

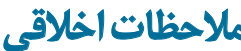 \\ يييروى از اصول اخلاق يثوهش}

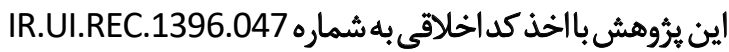

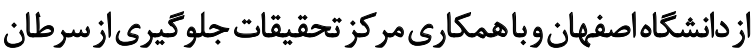

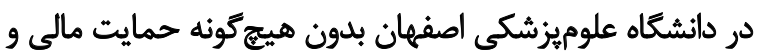

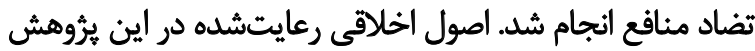

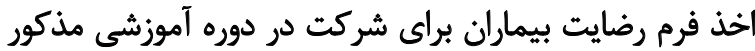

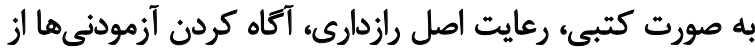

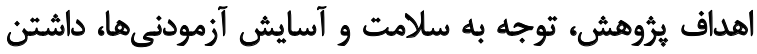

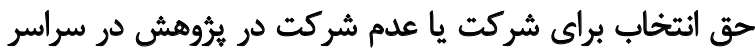

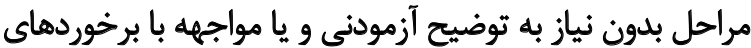

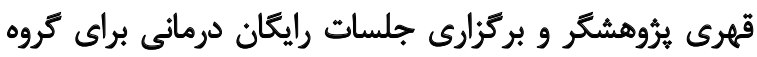

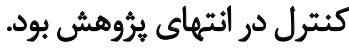

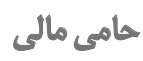

اين مقاله از رساله دكترى نويسنده دوم، سيد على كلاهدئ داندوزان،

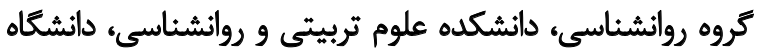
اصفهان است و حامى مالى ندارد.

$$
\text { مشاركت ثويسند مَان }
$$

مفهومسازى، روششناسى، اعتبارسنجى، تحليل، تحقيق

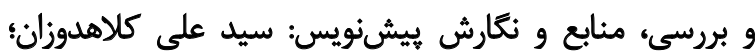

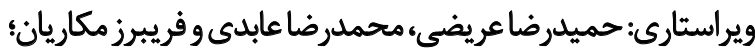

نهايىسازى نوشته، بصرى سازى و نظارت: محمدباقر كجبارياف.

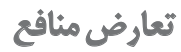

بنابه|ظهارات نويسندكان اين مقاله هيجّونه تعارض منافعى ندارد..

$$
\text { تشكرو وقدوراني }
$$

همجينين بدينوسيله از مديريت و كاركنان محترم بيمارستان

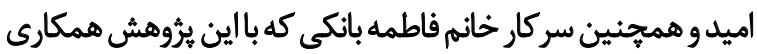
و همراهى فراوانى داشتهاند تشكر و قدردانى به عمل مي آيد.

\section{نتيجلكيرى}

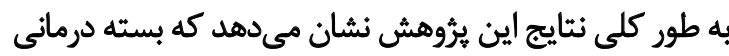

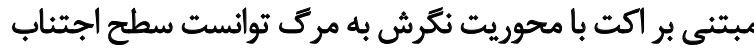

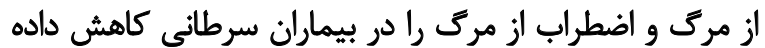

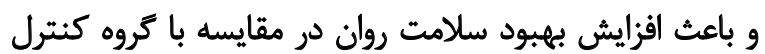

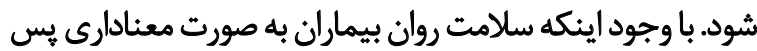

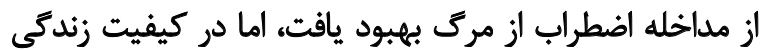

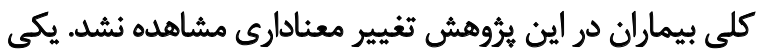

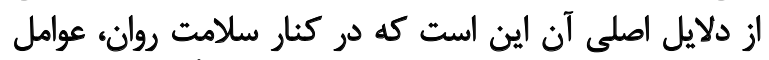

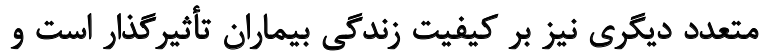

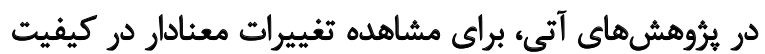

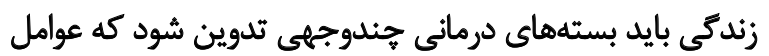

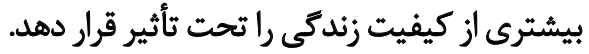

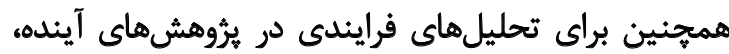

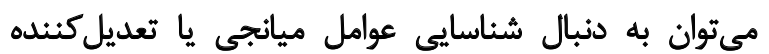

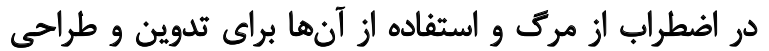

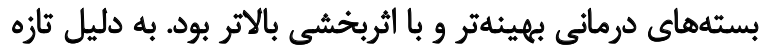

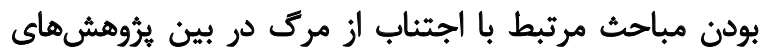

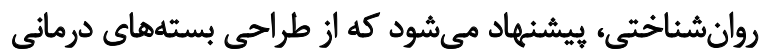

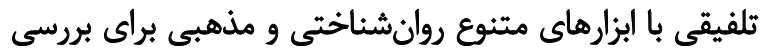

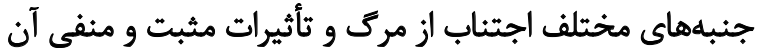

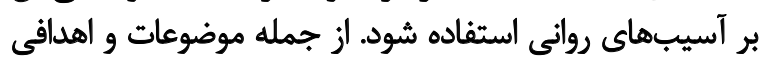

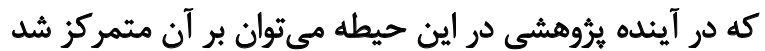

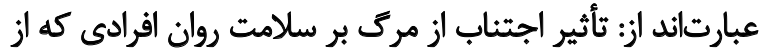

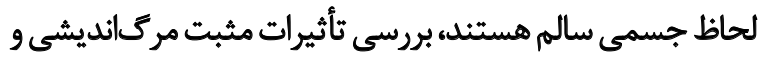

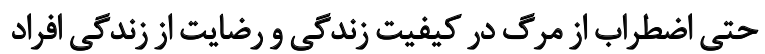

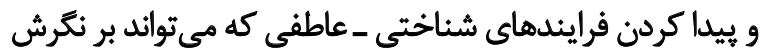
انسانها به مقوله مرك ثأثيركذار باشد.

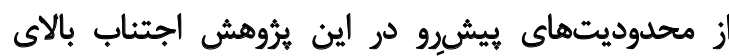

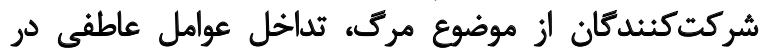

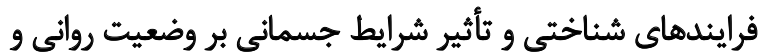

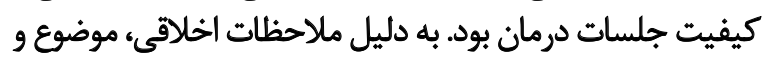

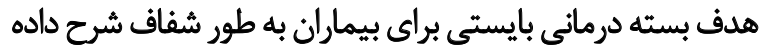

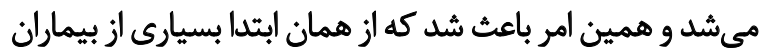

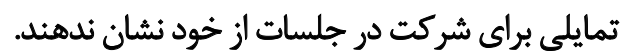

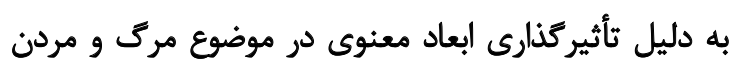

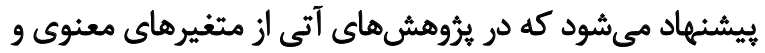

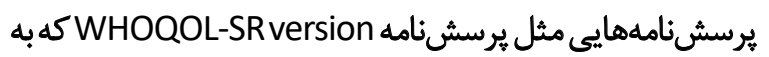

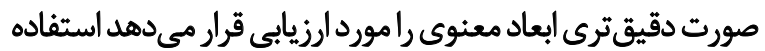

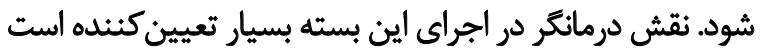

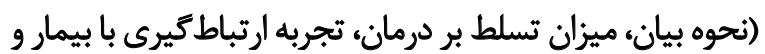

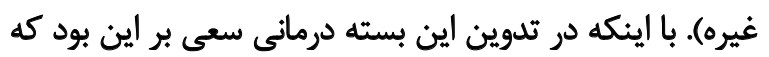




\section{References}

[1] Abachizadeh K, Keramatinia AA. [Anticipating cancer rates of Iran in 2025 (Persian)]. Community Health. 2016; 3(1):66-73. http://ojs2.sbmu.ac.ir/ch/article/view/13101

[2] Stone P, Hardy J, Broadley K, Tookman AJ, Kurowska A, A'Hern R. Fatigue in advanced cancer: A prospective controlled crosssectional study. British Journal of Cancer.1999; 79(9-10):1479-86. [DOI:10.1038/sj.bjc.6690236] [PMID] [PMCID]

[3] Walsh D, Donnelly S, Rybicki L. The symptoms of advanced cancer: Relationship to age, gender, and performance status in 1,000 patients. Supportive Care in Cancer. 2000; 8(3):175-9. [DOI:10.1007/s005200050281] [PMID]

[4] Grabsch B, Clarke DM, Love A, McKenzie DP, Snyder RD, Bloch S, et al. Psychological morbidity and quality of life in women with advanced breast cancer: A cross-sectional survey. Palliative \& Supportive Care. 2006; 4(1):47-56. [DOI:10.1017/ S1478951506060068] [PMID]

[5] Kissane DW, Love A, Hatton A, Bloch S, Smith G, Clarke DM, et al. Effect of cognitive-existential group therapy on survival in early-stage breast cancer. Journal of Clinical Oncology. 2004; 22(21):4255-60. [DOI:10.1200/JCO.2004.12.129] [PMID]

[6] Kugaya A, Akechi T, Okuyama T, Nakano T, Mikami I, Okamura $\mathrm{H}$, et al. Prevalence, predictive factors, and screening for psychologic distress in patients with newly diagnosed head and neck cancer. Cancer. 2000; 88(12):2817-23. [DOI:10.1002/10970142(20000615)88:12<2817::aid-cncr22>3.0.co;2-n] [PMID]

[7] Vehling S, Lehmann C, Oechsle K, Bokemeyer C, Krüll A, Koch $\mathrm{U}$, et al. Is advanced cancer associated with demoralization and lower global meaning? The role of tumor stage and physical problems in explaining existential distress in cancer patients. PsychoOncology. 2012; 21(1):54-63. [DOI:10.1002/pon.1866] [PMID]

[8] Wilson KG, Chochinov HM, Skirko MG, Allard P, Chary S, Gagnon PR, et al. Depression and anxiety disorders in palliative cancer care. Journal of Pain and Symptom Management. 2007; 33(2):118-29. [DOI:10.1016/j.jpainsymman.2006.07.016] [PMID]

[9] Coyne JC, Stefanek M, Palmer SC. Psychotherapy and survival in cancer: The conflict between hope and evidence. Psychological Bulletin. 2007; 133(3):367-94. [DOI:10.1037/0033-2909.133.3.367] [PMID]

[10] El Sayed Fadila D, Abd El-Sattar Ebeid I, El-Gilany AH. Death attitude and its associated factors in institutionalized elderly: A cross-section study in North Eastern Delta, Egypt. Journal of Nursing and Health Science. 2018; 7(6):12-9. https://www.researchgate.net/publication/339484844

[11] Salajegheh S, Raghibi M. [The effect of combined therapy of spiritual-cognitive group therapy on death anxiety in patients with cancer (Persian)]. Journal of Shahid Sadoughi University of Medical Sciences. 2014; 22(2):1130-9. http:/ / jssu.ssu.ac.ir/article1-2322-en.html

[12] Hayes SC, Strosahl KD, editors. A practical guide to acceptance and commitment therapy. New York: Springer Science and Business Media Inc; 2010. https://books.google.com/ books?id=rpKLcgAACAAJ\&dq

[13] Törneke N. Learning RFT: An introduction to relational frame theory and its clinical application. Oakland, CA: New
Harbinger Publicaions; 2010. https://books.google.com/ books?id=PYRy9a3iLtEC\&dq

[14] Ando M, Morita T, Lee V, Okamoto T. A pilot study of transformation, attributed meanings to the illness, and spiritual well-being for terminally ill cancer patients. Palliative \& Supportive Care. 2008; 6(4):335-40. [DOI:10.1017/S1478951508000539] [PMID]

[15] Helgeson VS, Reynolds KA, Tomich PL. A meta-analytic review of benefit finding and growth. Journal of Consulting and Clinical Psychology. 2006; 74(5):797-816. [DOI:10.1037/0022 006X.74.5.797] [PMID]

[16] Taylor EJ. Transformation of tragedy among women surviving breast cancer. Oncology Nursing Forum. 2000; 27(5):781-8. [PMID]

[17] Ciarrochi J, Fisher D, Lane L. The link between value motives, value success, and well-being among people diagnosed with cancer. Psycho-Oncology. 2011; 20(11):1184-92. [DOI:10.1002/ pon.1832] [PMID]

[18] Feros DL, Lane L, Ciarrochi J, Blackledge JT. Acceptance and Commitment Therapy (ACT) for improving the lives of cancer patients: A preliminary study. Psycho-Oncology. 2013; 22(2):45964. [DOI:10.1002/pon.2083] [PMID]

[19] Hayes SC, Villatte M, Levin M, Hildebrandt M. Open, aware, and active: Contextual approaches as an emerging trend in the behavioral and cognitive therapies. Annual Review of Clinical Psychology. 2011; 7:141-68. [DOI:10.1146/annurevclinpsy-032210-104449] [PMID]

[20] Hayes SC, Strosahl KD, Wilson KG. Acceptance and commitment therapy: The process and practice of mindful change. $2^{\text {nd }} \mathrm{ed}$. New York: Guildford Press; 2012. https://books.google.com/ books?id=5_xAm0VF4X8C\&source

[21] Spiegler MD, Guevremont DC. Contemporary behavior therapy. $5^{\text {th }}$ ed. Belmont, CA: Wadsworth; 2010. https:/ / books.google. com/books?id=iwauXH0eItoC\&dq

[22] Mirzaeidoostan Z, Zargar Y, Zandi Payam A. [The effectiveness of acceptance and commitment therapy on death anxiety and mental health in women with HIV in Abadan City, Iran (Persian)]. Iranian Journal of Psychiatry and Clinical Psychology. 2019; 25(1):2-13. [DOI:10.32598/ijpcp.25.1.2]

[23] Mohebat Bahar S, Akbari MS, Maleki F, Moradi Joo M. [The effectiveness of group psychotherapy based on acceptance and commitment on quality of life in women with breast cancer (Persian)]. Thoughts and Behavior in Clinical Psychology. 2015; 9(34):17-26. https://jtbcp.riau.ac.ir/article_104.html

[24] Sobouhi R, Fatehizade MA, Ahmadi SA, Etemadi O. [The ef fect of counseiing-based on Acceptance and Commitment Therapy (ACT) on the quality of life of women attending the cultura centers of Isfahan (Persian)]. Shenakht Journal of Psychology and Psychiatry. 2015; 2(1):42-53. http://shenakht.muk.ac.ir/article1-89-en.html

[25] Irandoos F, Taher Neshatdoost H, Nadi MA. [The effectiveness of Acceptance and Commitment Therapy (ACT) on quality of life of women with chronic low back pain (Persian)]. International Journal of Behavioral Sciences. 2014; 8(1):89-96. magiran.com/ p1274153

[26] Ghomian S, Shairi MR. The effectiveness of Acceptance and Commitment Therapy for children with chronic pain on the quality of life on 7 to 12 year-old children. International Journal of Pediatrics. 2014; 2(3-2):47-55. [DOI:10.22038/IJP.2014.2995] 
[27] Rafiee M, Sedrpoushan N, Abedi MR. Study and investigate the effect of Acceptance and Commitment Therapy on reducing anxiety symptoms and body image dissatisfaction in obese. Journal of Social Welfare and Human Rights. 2013; 1(2):13-8. http:// jswhr.com/vol-1-no-2-december-2013-abstract-3-jswhr

[28] Iravani Sh, Kajbafnezhad H. Effectiveness of Acceptance and Commitment Therapy on anxiety in patients with heart disease. International Journal of Biology, Pharmacy and Allied Sciences. 2015; 4(10):84-98. https://ijbpas.com/pdf/2015/ October/1441634901MS\% 20IJBPAS\% 202015\%20OCT\%20 SPCL\%201010.pdf

[29] Molavi P, Mikaeili N, Rahimi N, Mehri S. [The effectiveness of Acceptance and Commitment Therapy based on reducing anxiety and depression in students with social phobia (Persian)]. Journal of Ardabil University of Medical Sciences. 2014; 14(4):412-23. http://jarums.arums.ac.ir/article-1-745-en.html

[30] González-Fernández S, Fernández-Rodríguez C, Paz-Caballero MD, Pérez-Álvarez M. Treating anxiety and depression of cancer survivors: Behavioral activation versus acceptance and commitment therapy. Psicothema 2018; 30(1):14-20. [DOI:10.7334/psicothema2017.396] [PMID]

[31] RajabiS, Yazdekhasti F. [The effectiveness of the acceptance and commitment group therapy on anxiety and depression in women with MS who were referred to the MS association (Persian)]. Journal of Clinical Psychology. 2014; 6(1):29-38. [DOI:10.22075/ JCP.2017.2152]

[32] Dehghani Najvani B, Taher Neshatdoost H, Abedi MR, Mokarian F. The effect of Acceptance and Commitment Therapy on depression and psychological flexibility in women with breast cancer. Zahedan Journal of Research in Medical Sciences. 2015; 17(4):e965. [DOI:10.5812/zjrms.17(4)2015.965]

[33] Tamannaeifar Sh, Gharraee B, Birashk B, Habibi M. A comparative effectiveness of Acceptance and Commitment Therapy and group cognitive therapy for major depressive disorder. Zahedan Journal of Research in Medical Sciences. 2014; 16(10(Suppl)):60-3. https://sites.kowsarpub.com/zjrms/articles/1309.html

[34] Hor M, Aghaie A, Abedi A, Attari A. [The effectiveness of Acceptance and Commitment Therapy on depression in patients with type 2 diabetes (Persian)]. Journal of Research in Behavioral Sciences. 2013; 11(2):121-8. http://rbs.mui.ac.ir/article-1-305-en. $\mathrm{html}$

[35] Amin Pur R, Ghorbani M. [The effectiveness of Acceptance and Commitment Therapy on stress coping strategies in women with ulcerative colitis (Persian)]. Govaresh. 2015; 20(1):34-42. http:// www.govaresh.org/index.php/dd/article/view/1351

[36] Nasiri A, Kazemi-Zahrani H. The effectiveness of group acceptance and commitment therapy on pain intensity, pain catastrophizing and pain-associated anxiety in patients with chronic pain. Asian Social Science. 2015; 11(26):112-20. [DOI:10.5539/ass. v11n26p112]

[37] Walker J, Hansen CH, Butcher I, Sharma N, Wall L, Murray G, et al. Thoughts of death and suicide reported by cancer patients who endorsed the "suicidal thoughts" item of the PHQ-9 during routine screening for depression. Psychosomatics. 2011; 52(5):4247. [DOI:10.1016/j.psym.2011.02.003] [PMID]

[38] Brudek P, Sękowski M, Steuden S. Polish adaptation of the death attitude profile-revised. OMEGA - Journal of Death and Dying. 2020; 81(1):18-36. [DOI:10.1177/0030222818754670]
[39] Peters L, Cant R, Payne S, O'Connor M, McDermott F, Hood $\mathrm{K}$, et al. How death anxiety impacts nurses' caring for patients at the end of life: A review of literature. The Open Nursing Journal. 2013; 7:14-21. [DOI:10.2174/1874434601307010014] [PMID] [PMCID]

[40] Kashdan TB, Barrios V, Forsyth JP, Steger MF. Experiential avoidance as a generalized psychological vulnerability: Comparisons with coping and emotion regulation strategies. Behaviour Research and Therapy. 2006; 44(9):1301-20. [DOI:10.1016/j. brat.2005.10.003] [PMID]

[41] Tull MT, Roemer L. Emotion regulation difficulties associated with the experience of uncured panic attacks: Evidence of experiential avoidance, emotional nonacceptance, and decreased emotional clarity. Behavior Therapy. 2007; 38(4):378-91. [DOI:10.1016/j.beth.2006.10.006] [PMID]

[42] Carver CS, Pozo C, Harris SD, Noriega V, Scheier MF, Robinson DS, et al. How coping mediates the effect of optimism on distress: A study of women with early-stage breast-cancer. Journal of Personality and Social Psychology. 1993; 65(2):375-90. [DOI:10.1037/0022-3514.65.2.375] [PMID]

[43] Pennebaker JW, Seagal JD. Forming a story: The health benefits of narrative. Journal of Clinical Psychology. 1999; 55(10):124354. [DOI:10.1002/(SICI)1097-4679(199910)55:10<1243::AIDJCLP6>3.0.CO;2-N]

[44] Nejat S, Montazeri A, Holakouie Naieni K, Mohammad K, Majdzadeh SR. [The World Health Organization quality of Life (WHOQOL-BREF) questionnaire: Translation and validation study of the Iranian version (Persian)]. Journal of School of Public Health and Institute of Public Health Research. 2006; 4(4):1-12. http://sjsph.tums.ac.ir/article-1-187-en.html

[45] Wong PTP, Reker GT, Gesser G. (1994). Death attitude profilerevised: A multidimensional measure of attitudes toward death. In: Neimeyer RA, editor. Series in Death Education, Aging, and Health Care. Death Anxiety Handbook: Research, Instrumentation, and Application. Abingdon: Taylor \& Francis. pp. 121-48. https:/ / psycnet.apa.org/record/1994-97098-006

[46] Basharpoor S, Vojodi B, Atarod N. [The relation of religious orientation and attitudes toward death with quality of life and somatization symptoms in women (Persian)]. Health Psychology. 2014; 3(10):80-97. http://hpj.journals.pnu.ac.ir/article_986.html

[47] Hayes SC, Strosahl K, Wilson KG, Bissett RT, Pistorello J, Toarmino D, et al. Measuring experiential avoidance: A preliminary test on a working model. The Psychological Record. 2004; 54(4):553-78. [DOI:10.1007/BF03395492]

[48] Bond FW, Hayes SC, Baer RA, Carpenter KM, Guenole N, Orcutt HK, et al. Preliminary psychometric properties of the Acceptance and Action Questionnaire-II: A revised measure of psychological inflexibility and experiential avoidance. Behavior Therapy. 2011; 42(4):676-88. [DOI:10.1016/j.beth.2011.03.007] [PMID]

[49] Almasi Z, Rafiemanesh H, Salehiniya H. Epidemiology characteristics and trends of incidence and morphology of stomach cancer in Iran. Asian Pacific Journal of Cancer Prevention. 2015; 16(7):2757-61. [DOI:10.7314/APJCP.2015.16.7.2757] [PMID]

[50] Dolatkhah R, Somi MH, Asvadi Kermani I, Ghojazadeh M, Asghari Jafarabadi M, Farassati F, et al. (2015). Increased colorectal cancer incidence in Iran: A systematic review and meta-analysis. BMC Public Health. 2015; 15:997. [DOI:10.1186/s12889-015-23429] [PMID] [PMCID] 
[51] Politi MC, Enright TM, Weihs KL. The effects of age and emotional acceptance on distress among breast cancer patients. Supportive Care in Cancer. 2007; 15(1):73-9. [DOI:10.1007/s00520006-0098-6] [PMID]

[52] Stanton AL, Danoff-Burg Sh, Huggins ME. The first year after breast cancer diagnosis: Hope and coping strategies as predictors of adjustment. Psycho-Oncology. 2002; 11(2):93-102. [DOI:10.1002/pon.574] [PMID]

[53] Angiola JE, Bowen AM. Quality of life in advanced cancer: An acceptance and commitment therapy view. The Counseling Psychologist. 2013; 41(2):313-35. [DOI:10.1177/0011000012461955] 
This Page Intentionally Left Blank 